\title{
giFORSETI
}

\section{¿Regla de subcapitalización o earnings stripping rule?: Análisis evolutivo y comparativo de las normas que limitan la deducción de gastos financieros y su enfoque en el Perú}

\author{
Raúl Alfredo Campos Ramírez* \\ José Julio Hernández Morales**
}

Resumen. - En el 2018, se produjo una de las reformas tributarias más importantes en los últimos años $\mathrm{y}$, como parte de tal reforma, se modificó el conocido régimen de subcapitalización del artículo 37.a de la Ley del Impuesto a la Renta. De esta manera, en el presente trabajo, buscamos desentrañar los fundamentos y antecedentes de esta norma antielusiva, así como sus características y falencias de acuerdo con el panorama jurídico y económico que atraviesa el país.

\begin{abstract}
In 2018 one of the most important tax reforms in recent years took place. As part of this reform, the well-known thin capitalization regime stipulated in Article 37 of the Income Tax Law was modified. Therefore, in this paper, we aim to unravel the foundations and antecedents of this anti-avoidance rule, as well as its characteristics and shortcomings according to the legal and economic situation that the country is going through.
\end{abstract}

Palabras claves. - Deducción - gastos financieros - earnings stripping rules - OCDE endeudamiento - EBITDA - grupos multinacionales - subcapitalización - préstamos intereses - antielusivo - erosión - base imponible - patrimonio - BEPS - MNEs - profit shifting - ratio.

Keywords. - Deduction - financial expenses - earnings stripping rules - OECD - debt EBITDA - multinational groups - thin capitalization - loans - interest - anti-avoidance erosion - tax base - equity - BEPS - MNEs - profit shifting, ratio.

* Abogado por la Pontificia Universidad Católica del Perú (PUCP). Senior Associate en el área de Tax Consulting de PricewaterhouseCoopers Perú (PWC). Se ha desempeñado como Jefe de Prácticas del curso de Derecho Tributario II en la Facultad de Derecho de la Universidad del Pacífico.

** Estudiante de derecho de décimo segundo ciclo de la Universidad de Piura (UDEP). Practicante Pre-Profesional en el área de Tax Consulting de PricewaterhouseCoopers Perú (PWC).

*** Agradecemos a Katarzyna Dunin-Borkowski y Ronald Vargas por su valioso aporte en la redacción del presente artículo. 


\section{Introducción}

La Ley 27356, vigente a partir del 1 de enero de 2001 incorporó por primera vez la regla de subcapitalización al ordenamiento jurídico peruano. Esta regla estableció un límite a la deducción del gasto de intereses provenientes de operaciones entre empresas vinculadas: solo podían deducir intereses por un monto de endeudamiento de hasta 3 veces el patrimonio neto de la empresa.

El objetivo de la norma era claro. El Estado buscaba evitar que una empresa abuse de la deducción de gasto financiero a través de financiamientos otorgados por empresas del mismo grupo con el único propósito de reducir la base imponible.

Dicha norma permaneció, en esencia, invariable por casi dos décadas. Sin embargo, a raíz de los lineamientos emitidos por la OECD, puntualmente, la Acción 4 del Plan BEPS1, el Gobierno modificó la regla de subcapitalización. Los cambios fueron introducidos por el Decreto Legislativo 1424 (D.L. 1424).

El referido decreto introdujo una modificación gradual del régimen. En primer lugar, estableció un régimen transitorio para los años 2019 y 2020. La principal y relevante - modificación consistió en ampliar la aplicación de la norma a todos los préstamos, sin distinguir si fueron otorgados por empresas vinculadas o por terceros no vinculados. En segundo lugar, se estableció un régimen que, salvo cambio legislativo, entrará en vigencia el 1 de enero de 2021. Este último supone un gran cambio en el modo de calcular la limitación a la deducción de intereses: solo se podrán deducir los intereses que no superen el 30\% del EBITDA2 del ejercicio anterior.

Desde la publicación del D.L. 1424 la reforma normativa ha sido materia de críticas de todo tipo. Principalmente, los comentarios se orientan a criticar que el Gobierno peruano ha adoptado las recomendaciones de la OECD plasmadas en el Reporte Final de la Acción 4 de BEPS, sin tomar en consideración la realidad económica y jurídica de nuestro país.

Bajo este contexto, el objetivo principal de este trabajo es analizar los conceptos que están detrás de las recomendaciones de la OECD, así como de la modificación de la norma de subcapitalización peruana. Justamente, a partir de dicho marco conceptual se evaluará si tales modificaciones son pertinentes o no; y, si causan más perjuicios que beneficios.

Con este propósito, se partirá analizando - y diferenciando - los conceptos de subcapitalización y earnings stripping3, siendo este último el problema que busca combatir la Acción 4 del Plan BEPS. En segundo lugar, describiremos la evolución

1 OECD. Limiting Base Erosion Involving Interest Deductions and Other Financial Payments, Action 4 2016 Update: Inclusive Framework on BEPS. París: OECD Publishing, 2017.

2 Se debe entender por EBITDA a la renta neta luego de efectuada la compensación de pérdidas más los intereses netos, depreciación y amortización.

3 Desde nuestro punto de vista, la OCDE se refiere a este término como base erosion and profit shifting (BEPS). 
histórica de los mecanismos que combaten ambos fenómenos, analizando el contexto y problemática que suscitó su evolución legislativa.

Una vez desarrollado el marco conceptual, evaluaremos la idoneidad de adoptar los lineamientos de la OECD en un contexto como el nuestro. Posteriormente a ello, explicaremos cómo, la falta de un análisis profundo, no solo de la Acción 4 del Plan BEPS, sino en general del funcionamiento y utilidad de los mecanismos para enfrentar la subcapitalización y el profit shifting generan diversas problemáticas que requieren ser corregidas. Finalmente, haremos énfasis en cómo la crisis suscitada por el Covid-19 dejan al descubierto las graves falencias de la nueva norma.

\section{La subcapitalización y Earnings Stripping: desencuentros y mecanismos de combate}

\section{i. El problema de la subcapitalización}

Como punto de partida, es importante tomar en cuenta que la subcapitalización proviene de las ciencias económicas y empresariales. Por lo mismo, este concepto ha sido analizado - en primera medida - por el derecho mercantil, antes que por el derecho tributario.

Según el profesor PAZ ARES la subcapitalización es “[c]ualquier desproporción conmensurable- $\mathrm{o}$, si se prefiere, inequívocamente constatable- entre la magnitud del capital (...) y el nivel de riesgo de la empresa que en cada caso se programe para llevar a efecto el objeto social4". Dicho de otro modo, una sociedad se encuentra subcapitalizada cuando sus socios no han realizado los aportes (fondos) necesarios para desarrollar su objeto social5.

Según la definición mercantil de este fenómeno, es posible distinguir dos tipos de subcapitalización: (a) material y (b) nominal. La primera hace referencia, justamente, al hecho que una empresa no cuente con los recursos suficientes para llevar a cabo su objeto social. La segunda, en cambio, se produce cuando las sociedades tienen un capital social insuficiente; sin embargo, estas necesidades son cubiertas por los socios a través de créditos y no mediante aportes de capital6. Y, por lo mismo, existe una preponderancia de la deuda (préstamo) frente al patrimonio (capital).

4 GUASCH MARTORELL, Rafael. “La doctrina de la infracapitalización: aproximación conceptual a la infracapitalización de las sociedades". En Revista de Derecho Mercantil, núm. 234, 1999, pp. 1489-1514.

5 SÁNCHEZ RUS, Heliodoro. El capital social: presente y futuro (tesis doctoral). Barcelona: Universidad Pompeu Fabra, 2011, p. 364.

6 GUASCH MARTORELL, Rafael. “La doctrina de la infracapitalización: aproximación conceptual a la infracapitalización de las sociedades". En Revista de Derecho Mercantil, núm. 234, 1999, pp. 1489-1514. 
Así pues, a pesar de ser un concepto propio de las ciencias económicas, la subcapitalización - nominal - tiene especial relevancia dentro del derecho tributario.

Esto se debe, principalmente, a la diferencia en el tratamiento tributario - y a las ventajas que se pueden obtenerse de él - de un aporte de capital y un préstamo. En efecto, en un préstamo, la retribución percibida por el préstamo es el pago de intereses. En cambio, en el caso de un aporte de capital, el accionista recibirá dividendos (o retiro de utilidades).

Como consecuencia de esta diferencia, la gran mayoría de jurisdicciones del mundo otorga diferentes efectos tributarios a cada uno7. Así, por regla general, el pago de intereses es un gasto deducible en la determinación del IR8; por consiguiente, supone una reducción de la base imponible del tributo. En contraste, generalmente el pago de dividendos no solo no es deducible, sino que implica la previa declaración de la utilidad tributaria, que es gravada con el IR9.

En ese sentido, cuando una empresa se encuentra subcapitalizada (i.e. tenga una desproporción entre el capital social y el nivel de financiamiento), en lugar de reconocer una utilidad - y posteriormente pagar dividendos - reducirá su base imponible a través del pago de intereses. Cuando este fenómeno se produce a gran escala, inevitablemente, la recaudación de un país se verá afectada. Precisamente, las primeras reglas de subcapitalización se originaron para combatir las estructuras excesivamente apalancadas, las cuales privaban a los gobiernos de los ingresos fiscales necesariosio.

De lo anterior, tenemos que, para el derecho tributario11, la subcapitalización es “aquella situación en la que la prevalencia de los recursos ajenos [financiamiento

7 Precisamente, dado que el origen del problema ocasionado por la subcapitalización es la diferencia entre el tratamiento tributario de los intereses de dividendos, se han propuesto medidas que eliminen o difuminen esta diferencia. Específicamente, se han planteado mecanismos: (a) Allowance for corporate equity ("ACE") en virtud del cual prevé que un porcentaje del rendimiento del capital (i.e., dividendos) sea deducible de las ganancias corporativas. Los países que han introducido medidas de este estilo son Italia, Brasil, Bélgica, entre otros; y, (b) Comprehensive business income tax ("CBIT"), en virtud del cual se deshabilita la posibilidad de deducir los pagos de gastos financiero, eliminando el trato fiscal preferente a favor de financiamiento vía préstamos. En estricto, ningún país ha introducido una medida de este estilo, aunque las earnings stripping rules comparten algunas características. Los autores DE MOOIJ, Ruud A. y DEVEREUX, Michael P. analizan en detalle estas propuestas en su trabajo, denominado "Alternative Systems of Business Tax in Europe. An applied analysis of ACE and CBIT Reforms", Taxation Papers 17, Directorate General Taxation and Customs Union, Comisión Europea.

8 Precisamente, el artículo 37 (inciso a) de la Ley del IR establece que, "son deducibles los intereses deudas y los gastos originados por la constitución, renovación o cancelación de las mismas (...)”.

9 AGUILAR SALDIVAR, Lourdes. "El tratamiento tributario de la subcapitalización en la legislación peruana y en la legislación comparada". En Ius et Veritas, núm. 23, 2001, p. 248.

10 Traducción propia de: "Thin capitalization rules originated in those countries to combat excessively leveraged financing structures, which deprived governments of needed tax revenue".

WEBBER, Stuart. "Thin Capitalization and Interest Deduction Regulations". Discussion Paper No. 8. Copenhague, Dinamarca: Copenhagen Group on International Taxation - CORIT, p. 9.

11 Según la autora, la subcapitalización es una situación en la que una empresa disfraza la entrega de fondos propios (instrumentos de capital) vía un endeudamiento (instrumentos de deuda) con 
externo] sobre los fondos propios [financiamiento interno] en la financiación de una sociedad supone una pérdida recaudatoria para la Hacienda Pública12".

Sin perjuicio de lo anterior, resulta necesario dejar en claro que, la subcapitalización (tributaria) resulta un problema para el fisco, principalmente, cuando la matriz (prestamista) y la subsidiaria (prestataria) están domiciliados en jurisdicciones distintas.

En este sentido, cuando ambas empresas están domiciliadas en el mismo país el efecto tributario se diluye. Así, mientras la subsidiaria deduce los intereses de su base imponible pagando un menor impuesto a la renta ("IR"), los intereses pagados, serán recibidos por la matriz, quien terminará incorporando los intereses en su base imponible y, por tanto, pagará IR por tales ingresos. En consecuencia, aquello dejado de gravar en la subsidiaria, terminará siendo gravado en la matriz13.

\section{ii. El problema del earnings stripping}

Dentro del ámbito tributario, aparece el problema de la erosión de la base imponible y el traslado de beneficios (utilidades) o earnings stripping. Pues bien, se puede definir, de manera general, a este concepto como, la modalidad de planificación fiscal consistente en que una subsidiaria localizada en un país con una alta imposición efectúe pagos de excesivos montos de interés - deducible - a su matriz u a otra afiliada, domiciliada en un país con nula o baja imposición, con el objetivo de reducir la carga tributaria global de una MNE, erosionando la base imponible del impuesto a la renta de las sociedades pagadoras de los intereses que, de otro modo, verían su actividad económica gravada con un mayor impuesto14.

Así pues, la subcapitalización (en lo sucesivo, "SC") y el earnings stripping (en adelante, "ES") tienen en común que el efecto de ambos fenómenos es la erosión de la base imponible a través del excesivo pago de intereses. Sin embargo, como

la única finalidad de erosionar la base imponible mediante la deducción de intereses, a fin de determinar un menor impuesto a la renta por pagar.

DUNIN-BORKOWSKI, Katarzyna. ¿Debe mantenerse la norma de subcapitalización en la ley de impuesto a la renta peruana? (Tesis para optar al grado de Magíster). Lima: Pontificia Universidad Católica del Perú (PUCP), 2019, p. 39.

12 CENCERRADO MILLÁN, Emilio. “La Subcapitalización”. En CORDÓN EZQUERRO, Teodoro (dir.), Fiscalidad de los precios de transferencia (operaciones vinculadas). Madrid: Centro de Estudios Financieros, 2010, pp. 637-691.

13 Sin perjuicio de ello, es cierto que la subsidiaria deducirá un interés pagado que, no hubiera existido si se hubiera financiado mediante aportes de capital, en lugar, de préstamos.

14 Adaptación del siguiente fragmento: "A multinational enterprise ("MNE") may use cross-border earnings stripping as a tax planning strategy. This strategy involves a higher-tax affiliate making deductible payments to a low-or zero-tax affiliate to reduce the MNE's global effective tax rate and, in the process, erode the corporate tax bases of countries where its economic activity otherwise would be more highly taxed".

FLEMING JR., J. Clifton, PERONI, Robert y SHAY, Stephen. "Getting Serious About CrossBorder Earnings Stripping: Establishing an Analytical Framework", En North Carolina Law Review, vol. 93, 2015, p. 673. 
será desarrollado en las siguientes páginas el ES es un fenómeno que excede, por mucho, a la SC15.

Como punto de partida, debe quedar claro que, el ES es un fenómeno inherente a las empresas multinacionales ("MNEs" o "MNE" en caso individual).

De lo anterior, los autores reconocen que el referido problema se origina bajo dos premisas:

a) El principio de soberanía de los estados: si bien la economía se ha globalizado homogeneizando productos y precios en todo el mundo-, debido al mencionado principio, los estados fijan autónomamente las tasas, deducciones, entre otros criterios, para la determinación de la base imponible de sus tributos internos. Por este motivo, las tasas de los impuestos varían de país en país16.

b) Facilidad de las MNEs para trasladar sus actividades y activos: aprovechando su tamaño, estructura y presencia en distintas jurisdicciones, este tipo de empresas tienen "facilidad" para trasladar sus actividades de un país a otro. De igual manera, las MNEs puede trasladas sus activos (en especial los intangibles y financieros) de un país a otro. Por último, estas empresas pueden elegir libremente cómo financiar sus actividades; así pueden utilizar préstamos o aportes de capital a su elección.

Fluye de lo anterior que las MNEs aprovechan la mencionada facilidad para trasladar sus activos financieros para alocar sus ganancias a jurisdicciones con baja o nula imposición a través del otorgamiento de préstamos inter-compañía y, consiguientemente, una mayor deducción por pago de intereses.

De esta manera, se aprovecha la diferencia entre las tasas impositivas en los diferentes estados para obtener un mayor un ahorro fiscal. Puntualmente, el ahorro fiscal se traduce en una menor tasa impositiva efectiva del impuesto a la renta (o ganancias) que pagaría la MNE.

15 Los autores concuerdan que el earnings stripping puede presentarse sin estar conectado a una inversión corporativa, siendo que puede ejecutarse mediante el pago de regalías o el fee de servicios a una empresa que forma parte de la MNE. Sin perjuicio de ello, un Reporte del Departamento del Tesoro (Departament of the Treasury) de Estados Unidos, precisó que el uso de los préstamos entre partes vinculadas es el método más utilizado. Textualmente los autores refieren que, "(...) earnings stripping can take place without being connected with a corporate inversion, and earnings stripping can be accomplished by means of royalty and services fees payments in addition to interest payments. Nevertheless, the 2007 Treasury report on earnings stripping seemed to endorse the view that because (1) "the use of related-party debt arguably is the most readily available method of shifting income out of U.S. corporations,"54 (2) the "data on ... [inverted corporations] strongly suggests that these corporations are shifting substantially all of their income out of the United States, primarily through interest". Ibid., p. 691.

16 MASUI, Yoshihiro. “Interest Deduction, Corporate Groups and Tax Jurisdictions - A Hitchhiker's Guide to an Aspect of the BEPS Project". En Asia-Pacific Tax Bulletin March/April 2014, 2014, p. 103. 
Para efectos de que lo expuesto en los párrafos anteriores pueda ser comprendido de mejor manera, a continuación, describiremos dos escenarios prácticos de cómo las MNEs utilizan la deducción de intereses para reducir el pago de sus obligaciones fiscales. Hemos tomado ambos ejemplos del trabajo del profesor MASUI17.

\section{Escenario 1}

En este ejemplo, tenemos: (a) el Estado A con una alta imposición (30\%), (b) el Estado B con nula imposición (0\%), y (c) es un estado con una baja imposición $(15 \%)$.

En este escenario, tenemos que la MNE desarrolla sus actividades en el Estado C (baja imposición). Para financiar dichas operaciones, la compañía domiciliada en el Estado A obtiene un préstamo; y, a su vez, realiza un aporte de capital en la empresa domiciliada en el Estado C.

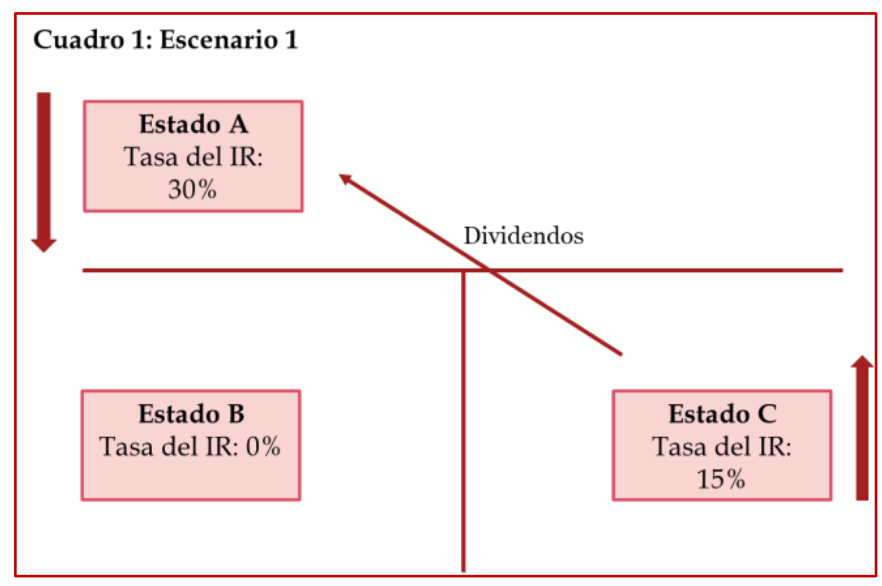

Fuente: MASUI (2014).

Los efectos tributarios son los siguientes:

a) La compañía domiciliada en el Estado A tiene un gasto deducible (i.e. interés pagado). De esta manera, reducirá su base imponible la cual está gravada con una tasa del $30 \%$.

b) La compañía en el Estado A, eventualmente, recibirá dividendos provenientes del Estado $\mathrm{C}$, los cuales si bien se incluirán en los ingresos (base), normalmente vienen con créditos de impuestos pagados en el extranjero, por lo que el impuesto finalmente pagado se ve reducido.

c) Los ingresos de la compañía del Estado C están gravados con una tasa del $15 \%$, que es mucho menor a la del Estado A.

Pues bien, supongamos que, en lugar de financiarse con capital, la compañía domiciliada en el Estado C opte por financiarse con un préstamo otorgado por la

$17 \quad$ Ibid., pp. 104-105. 
empresa del Estado A. Esta variación, eliminaría cualquier ventaja tributaria, conforme se aprecia a continuación:

a) La compañía domiciliada en el Estado A recibe el interés del Estado C, gravable en su integridad con una alta tasa del 30\% (no hay créditos tributarios que aplicar).

b) Si bien, la compañía $\mathrm{C}$ puede deducir los intereses, la deducción se realizará en un país con una baja tasa (esto es, una deducción menos efectiva).

Este simple ejemplo grafica las premisas explicadas previamente: (a) diferencias de las tasas impositivas (Estado A, B y C); y, (b) la facilidad de la MNEs para decidir dónde llevar a cabo sus actividades y, elegir el modo de financiación.

Es de notar que, en el mencionado ejemplo, no existe subcapitalización, por el contrario, los aportes de capital son más beneficiosos en el caso concreto.

\section{Escenario 2}

Igual que en el primer escenario, la MNE lleva a cabo sus actividades en el Estado C. Para financiar sus operaciones, la compañía establecida en el Estado A (alta imposición) obtiene un préstamo de terceros; $y$, realiza un aporte de capital en la sociedad establecida en el Estado B (nula imposición). Por su parte, la compañía domiciliada en el Estado $C$ (baja imposición) se financia a través de un préstamo de la empresa del Estado B.

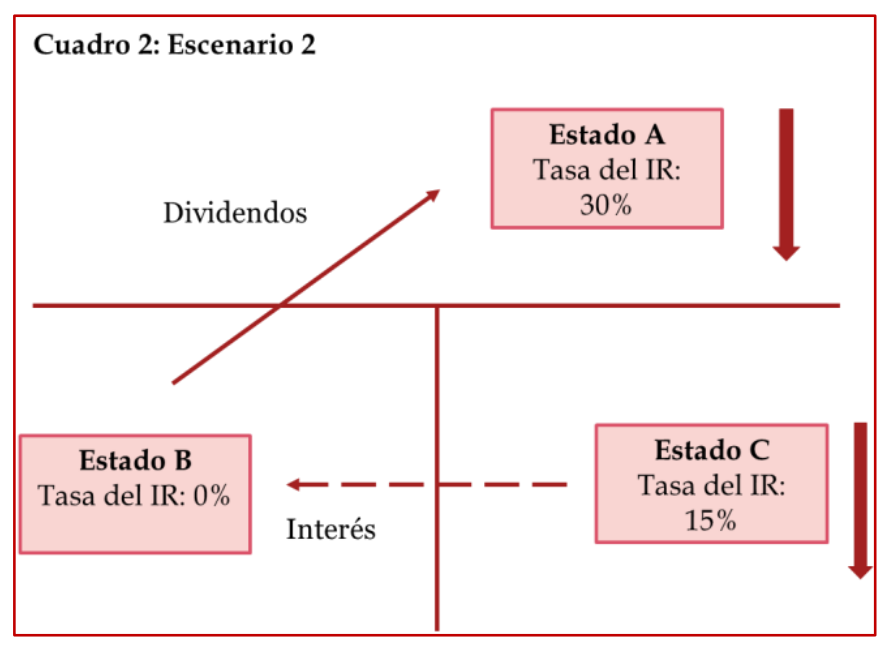

Fuente: MASUI (2014).

Los efectos tributarios son los siguientes:

a) La compañía del Estado A tiene un gasto deducible (i.e. interés pagado). Ello le permite reducir su base imponible que está gravada con una alta tasa.

b) La compañía del Estado A recibe los dividendos, los cuales estarán gravados; no obstante, la ganancia se neutraliza con la deducción de los intereses. 
¿Regla de subcapitalización o earnings stripping rule?: Análisis evolutivo y comparativo de las normas que limitan la deducción de gastos financieros y su enfoque en el Perú

c) La compañía del Estado B registra sus ingresos, pero no están gravados, debido a que es un país con nula imposición.

d) La compañía del Estado C deduce los intereses erosionando la base el IR del Estado C.

En este escenario, las ganancias son reconocidas en un país distinto al lugar de operación de la MNE (i.e., en el Estado B, a través del pago de intereses). La Administración Tributaria de los Estados A y C se verán perjudicas en dos formas: (a) gravarán un menor ingreso, y (b) se deducirán intereses en ambos estados.

En ambos casos se ejemplifica cómo las MNEs aprovechan: (a) su capacidad para trasladar sus activos (financieros) y actividades, así como para decidir cómo financiarse; y, (b) las distintas tasas impositivas de los estados; todo ello para erosionar la base imponible $\mathrm{y}$, trasladar sus beneficios a países con menores tasas de impuestos. Precisamente, dicho riesgo puede originarse en, al menos, tres escenarios18:

a) Las MNEs se financian con mayores niveles de préstamos de terceros en países con una tasa alta del IR.

b) Las MNEs utilizan préstamos intragrupo para generar deducciones de intereses superiores al gasto por interés real del grupo.

c) Las MNEs se financian con préstamos de terceros o intragrupo para generar ingresos exentos de impuestos.

\section{iii. Técnicas legislativas para combatir la subcapitalización y el earnings stripping}

En los dos apartados anteriores se ha distinguido que la SC y el ES son fenómenos diferentes. A pesar de ello, si bien, conceptualmente, la distinción es clara, como se desarrollará en el presente apartado, muchas veces esta diferencia no ha quedado plasmada en la legislación de los diferentes estados.

Precisamente, en este apartado se desarrollará la evolución de las técnicas legislativas que han adoptado los estados para combatir ambos fenómenos. Ello, a su vez, servirá para distinguir de mejor manera ambos conceptos.

En efecto, en una primera etapa, los estados combatían la SC sin contar con cláusulas específicas (esto es, a través del principio arm's length). Posteriormente, los estados incorporaron normas específicas para combatir dicho fenómeno (thin

18 Según la OECD, el riesgo de la erosión de la base imponible y el traslado de beneficios (BEPS) puede originarse en tres escenarios básicos: "(a) Groups placing higher levels of third party debt in high tax countries, (b) groups using intragroup loans to generate interest deductions in excess of group's actual third party interest expense; and, (c) groups using third party or intragroup financing to fund the generation of tax exempt income".

OECD. Limiting Base Erosion Involving Interest Deductions and Other Financial Payments, Action 4 2016 Update: Inclusive Framework on BEPS. París: OECD Publishing, 2017, p. 13. 
capitalization rules). Luego, los Estados ampliaron dichas cláusulas, incorporando elementos que, propiamente, no combaten la SC. Finalmente, y en especial con el apoyo de la OECD, los estados progresivamente han ido reemplazando las thin capitalization rules (en lo sucesivo, TCR) por normas del tipo earnings stripping (en adelante, ESR) 19 .

De lo anterior, es posible calificar todas estas herramientas legislativas de la siguiente manera20. Por un lado, tenemos las herramientas que combaten la subcapitalización: (a) enfoque subjetivo o enfoque arms' lenght y, (b) thin capitalization rules21. Y, por otro lado, están las normas del tipo earning stripping.

Esta división no es semántica, sino que responde, justamente, al problema que busca combatir cada una de ellas. Por este motivo, las distintas herramientas tienen un alcance y una estructura diferente22.

\section{a) Mecanismos para combatir la subcapitalización}

Como señalamos en el primer apartado, la subcapitalización supone una desproporción entre el capital y la deuda de una compañía. Precisamente, las empresas (especialmente, las MNEs) prefieren financiar sus subsidiarias con préstamos en lugar de aportes de capital para obtener un ahorro fiscal, a través de la deducción de intereses.

En ese sentido, las TCR tienen como objetivo combatir la mencionada desproporción. Con este propósito, las TCR limitan, para efectos de calcular los ingresos gravables con el IR, el monto de los préstamos que originan el gasto deducible por el pago de intereses.

\section{Enfoque subjetivo o "arm's length"}

El primer mecanismo consiste en, esencialmente, la aplicación del principio arm's length como lo realizó el Reino Unido hasta el año 201723.

19 También conocidas como interest limitation rules.

20 Esta calificación es similar a la realizada por las autoras DOURADO, Ana Paula y DE LA FERIA, Rita.

DOURADO, Ana Paula y DE LA FERIA, Rita. "Thin Capitalization Rules in the Context of the CCCTB". En LANG, M., et. al. (eds.), Common Consolidated Corporate Tax Base. Viena: Linde Verlag, 2008, p. 5.

21 También conocidas como safe harbor rules

22 Los autotres no hacen una diferencia tan tajante, pues consideran que las TCR (denominada por ellos como safe harbor rule) y las ESR son, propiamente, thin capitalization rules. Mientras que el enfoque arms's lenght sería un mecanismo distinto para combatir la subcapitalización.

RUF, Martin y SCHINDLER, Dirk. "Debt Shifting and Thin Capitalization Rules - German Experience and Alternative Approaches". En Nordic Tax Journal, núm. 1, 2015, p. 19.

23 Reino Unido ("RU”), siendo el único país que continúa aplicando este método. Aun así, cabe recalcar que, el RU tiene una larga historia en la implementación de normas de SC. En efecto, este país modificó sus normas de SC tres veces entre 1994 y 2004. No obstante, sus normas de SC fueron denunciadas ante la Corte Europea de Justicia (“ECJ”), quien determinó que su aplicación generaba un trato desigual entre las sociedades controladas por grupos nacionales (controlled local company) y las sociedades controladas por grupos extranjeros (controlled foreign 
En suma, este principio consiste en determinar si el préstamo de una empresa fue otorgado en las mismas condiciones en las que lo hubiera otorgado un prestamista independiente24.

Con este fin, la autoridad fiscal de cada estado debe realizar una evaluación ad hoc para determinar si: (a) la tasa de interés pagada corresponde a la tasa de interés que hubiera tenido que pagar a un tercero independiente, (b) el préstamo hubiera sido obtenido de un tercero independiente, en los mismos términos; $\mathrm{y}$, potencialmente si (c) la proporción entre deuda y capital de una compañía sería la misma, si los préstamos inter-compañía (i.e., entre partes vinculadas) hubieran estado restringidos. Si la operación (préstamo) no supera la evaluación, se impedirá la deducción de intereses que se originen de la misma.

De lo anterior, se puede apreciar que este enfoque tiene dos principales características: (a) su aplicación se restringe a préstamos entre partes vinculadas, pues, justamente, el baremo para su aplicación son los préstamos entre partes independientes; y, (b) la consecuencia de su aplicación es la limitación de la deducción de los intereses que se originen de dicha operación.

Precisamente, el fundamento para limitar la deducción es que, al no superar el test, se entiende que la operación realizada bajo la forma de un préstamo, en realidad camufla un aporte de capital. Por consiguiente, además de impedir la deducción de los intereses, la operación debería recibir el tratamiento jurídico de un aporte de capital (i.e., recategorización de la operación)25.

\section{Enfoque objetivo o thin capitalization rule}

En definitiva, los mecanismos subjetivos para combatir son ineficientes e ineficaces para combatir la subcapitalización, dado que requieren de grandes recursos y de

company). A partir de ello, concluyó que las normas de SC de RU violaban la cláusula de libertad de establecimiento previsto - actualmente - en el artículo 49 del Tratado de Funcionamiento de la Unión Europea. A partir de ese precedente, el RU viene aplicando el enfoque subjetivo para combatir la SC.

24 Al respecto, según esta autora, la aplicación de este enfoque consiste en evaluar en cada caso concreto verificando las operaciones efectuadas, a fin de determinar si el préstamo concedido corresponde a condiciones normales de mercado.

DUNIN-BORKOWSKI, Katarzyna. ¿Debe mantenerse la norma de subcapitalización en la ley de impuesto a la renta peruana? (Tesis para optar al grado de Magíster). Lima: Pontificia Universidad Católica del Perú (PUCP), 2019, p. 40.

25 La aplicación de este método tiene, al menos, dos ventajas: (a) su precisión, pues en la medida que el límite de intereses se calcula a partir de la deuda (préstamo) que la empresa está en capacidad de obtener (calculado a parir de un análisis independiente) y no a partir de un coeficiente o ratio abstracto, el monto es más exacto; y, (b) elimina el trato asimétrico entre empresas que formen parte de una MNE y aquellas que no.

OCDE (2012). Thin Capitalization Legislation a Background paper for a country tax administration (Plot Version for Comments), p. 9. Consulta realizada el 26 de julio de 2020. Disponible en: http://www.oecd.org/ctp/tax-global/5.\%20thin_capitalization_background.pdf 
una autoridad fiscal especializada para realizar las evaluaciones con precisión26. Por este motivo, los estados introdujeron normas específicas para combatir la SC.

Bajo este enfoque, el monto máximo de los préstamos cuyos intereses pueden ser deducidos se determinar aplicando un ratio predeterminado en la norma. Este ratio se establece a través de una comparación entre el endeudamiento de una compañía y su patrimonio (o capital)27. Por ejemplo, la norma puede permitir la deducción de los intereses provenientes de préstamos de hasta del triple del total de capital social de una sociedad (3/1). Los intereses que se originen de los préstamos que superen el ratio no serían deducibles28.

El uso de las TCR del tipo objetivo es el mecanismo más extendido para combatir el fenómeno de la subcapitalización29. Justamente, debido a la dispersión de estas cláusulas, su estructura, alcance y efectos no son uniformes en cada jurisdicción. Así, mientras que algunos países mantienen las características inherentes de una norma de SC, otros han ido incorporando elementos que son más propios de una norma del tipo de earnigns stripping.

Justamente, consideramos que la estructura y efectos de la TCR varía dependiendo de las siguientes cuatro variables:

a. Si el ratio de endeudamiento refleja el enfoque arm's length;

b. Si el ámbito de aplicación objetivo se restringe a los financiamientos entre entidades vinculadas o se extiende a aquellos otorgados por terceros;

c. Los efectos de la aplicación de la TCR; y,

d. Si se aplica sólo a las inversiones extranjeras o alcanza a las inversiones nacionales.

Respecto a la primera variable, por naturaleza, una TCR debe replicar un ratio de endeudamiento que presuma cuándo una empresa está subcapitalizada (cuente con una desproporción entre el endeudamiento y el patrimonio)30. Sin embargo,

26 Naturalmente, para que sea implementado con éxito se requiere grandes recursos, así como auditores con conocimientos especializados, capacitados para realizar una evaluación precisa. Ídem.

27 Justamente, la definición de qué se entiende por capital o patrimonio es uno de los grandes problemas en el diseño de una TCR.

28 OCDE (2012). Thin Capitalization Legislation a Background paper for a country tax administration (Plot Version for Comments), p. 12. Consulta realizada el 26 de julio de 2020. Disponible en: http://www.oecd.org/ctp/tax-global/5.\%20thin_capitalization_background.pdf

29 Según las autoras, hasta el año 2008 todos los países miembros de la Unión Europea (EU) que contaban con normas de SC, aplicaban el enfoque objetivo (i.e., ratio de endeudamiento contra patrimonio), con la excepción del Reino Unido que aplicaba el enfoque subjetivo.

DOURADO, Ana Paula y DE LA FERIA, Rita. "Thin Capitalization Rules in the Context of the CCCTB". En LANG, M., et. al. (eds.), Common Consolidated Corporate Tax Base. Viena: Linde Verlag, 2008.

$30 \quad \mathrm{Al}$ respecto, el autor señala que, “(...) resultará esencial determinar los criterios para identificar una situación en que la inyección de recursos se debió efectuar a través de aportes de capital en lugar de un préstamo”. Así pues, específicamente respecto a las TCR señala que, “(...) En este 
ciertamente esta labor es bastante complicada para los gobiernos, pues el nivel de endeudamiento depende, en gran medida, de cada sector económico. En ese sentido, si bien idealmente, los estados deberían prever ratios diferentes dependiendo del sector, debido a su dificultad y falta de practicidad, generalmente los estados incorporan un único ratio de endeudamiento.

En línea con lo anterior, reconociendo la dificultad de establecer un único ratio preciso, una TCR debe permitir que las empresas justifiquen su nivel de endeudamiento; en otras palabras, se debe admitir la prueba en contrario.

Naturalmente, la defensa de las empresas debe centrarse en demostrar que, a pesar de que su nivel de endeudamiento supera el ratio preestablecido en la norma, dichos créditos tienen un fundamento comercial válido; $y$, que se otorgaron en una situación de libre concurrencia (i.e., probar que se cumple con el principio arm's length).

De no incluir esta posibilidad, se impondría una presunción sin prueba en contrario (iure et de iure), la misma que se aleja de los fundamentos de una norma de subcapitalización.

En cuanto a la segunda variable, por naturaleza, una cláusula de subcapitalización debe estar destinada a la evaluación de los préstamos inter-compañía (entre vinculadas), pues, justamente, es en ese ámbito donde ocurre la subcapitalización31. No obstante, en distintos países las TCR restringen, incluso, los préstamos otorgados por terceros. En definitiva, esta característica es propia de una ESR, pues como detallamos previamente, el riesgo de earnings stripping puede presentarse incluso cuando las MNEs se financian con terceros en países con una alta imposición32.

Precisamente, en esta misma línea, además de estar restringidas a los préstamos "inter-compañía" las TCR de algunos países solo eran aplicables a los préstamos otorgados por sujetos (personas naturales o jurídicas) que tuvieran una participación mínima en la entidad prestataria. Por ejemplo, la cláusula (vigente

método la ley define a priori el coeficiente aplicable para determinar si la relación capital vs. pasivo expresa un sobreendeudamiento".

CHIRINOS SOTA, Carlos. "Redescubriendo realidades y formas. La norma Anti Subcapitalización como cláusula Antielusiva Especial”. En Derecho E Sociedad, núm. 31, pp. 295304.

31 Es de notar que, las empresas, eventualmente, podrían utilizar a terceros como intermediarios para camuflar préstamos entre vinculadas. Precisamente, este es uno de los motivos que justifican la ampliación del ámbito de aplicación de las TCR. Sin embargo, esto no quiere decir que los préstamos entre terceros generen, por naturaleza, un riesgo de subcapitalización. Siendo ello así, si la TCR de un país está restringida a los préstamos "inter-compañía", por aplicación de las cláusula anti-elusiva general podría aplicarse, incluso, a este tipo de esquemas, donde el tercero actúa, únicamente como intermediario $\mathrm{y}$, en realidad encubre un verdadero prestatario de la operación (el cual, si es una empresa vinculada).

32 Por ejemplo, hasta el 2018, la TCR peruana solo restringía la deducción de los préstamos otorgados por partes vinculadas; sin embargo, desde 2019 el alcance es a todos los financiamientos. 
hasta 2008) de Alemania exigía que el inversionista tuviera una participación mínima del 25\% en la sociedad para que la TCR fuera aplicable.

Ciertamente, esta es otra característica propia de las TCR, pues como se ha detallado previamente, la subcapitalización es, estricto, un problema entre accionistas (o matrices) y las subsidiarias o filiales.

Respecto a la tercera variable, las TCR pueden tener dos efectos: (a) recalificación de los préstamos que superen el ratio como aportes de capital; o, (b) simplemente limitar la deducción de estos intereses (i.e., considerarlos como gasto no deducible).

Ahora bien, por naturaleza, una TCR debería tener como efecto la recalificación del financiamiento como un aporte de capital y, con ello, gravar a los intereses como si se tratara de dividendos. Sin perjuicio de ello, en la mayoría de los países, incluyendo el Perú, únicamente, se restringe la deducción de los intereses33.

Finalmente, en cuanto a la última variable, originalmente, la aplicación de las TCR estaba restringida a las inversiones extranjeras (inbound). Si bien es una característica que no es inherente a la subcapitalización (como fenómeno societario), su inclusión demuestra que, para efectos tributarios, dicho fenómeno es un problema para las operaciones entre empresas domiciliadas en más de una jurisdicción, pues es ahí donde, precisamente, existe un riesgo para la recaudación de un país, ocasionado por la erosión de la base imponible de la empresa local, a través del traslado de los ingresos hacia jurisdicciones con nula o baja imposición34.

Conforme se puede apreciar, las TCR no son uniformes. En efecto, inicialmente las referidas cláusulas tenían las características inherentes de un mecanismo para combatir el fenómeno de la subcapitalización. Precisamente, esto se debe a que, los estados consideraban que, al combatir dicho fenómeno se combatiría eficazmente la erosión de la base imponible de las empresas locales y el traslado de sus beneficios (utilidades) a otras jurisdicciones.

Sin embargo, con el transcurso del tiempo los estados fueron ampliando la aplicación de las TCR, incorporando características que no respondían propiamente a una norma de subcapitalización. Justamente, ello responde a que, el problema que estaban combatiendo (i.e., earnings stripping) era más amplio que la subcapitalización y, por ello requería de ajustes.

33 Por ejemplo, en las TCR de Alemania (vigente hasta el 2008) y la de España (vigente hasta el 2012) la consecuencia de la aplicación de la TCR era la recategorización de los intereses como dividendos.

34 No obstante, el precedente Lankhorst-Hohorst de la Corte Europea de Justicia concluyó que, la TCR alemana -restringida a inversiones extranjeras- contravenía el principio de libertad de establecimiento (de la misma forma que las normas británicas). A partir de este hito, los estados tomaron tres caminos: (a) ampliar el ámbito de aplicación de las TCR a inversiones nacionales (e.g. Alemania), (b) restringir su ámbito de aplicación, para excluir las inversiones de países miembros de la Unión Europea (e.g. España), (c) derogar la TCR y aplicar únicamente el enfoque subjetivo (e.g. Reino Unido). 
¿Regla de subcapitalización o earnings stripping rule?: Análisis evolutivo y comparativo de las normas que limitan la deducción de gastos financieros y su enfoque en el Perú

\section{b) Técnicas legislativas para combatir el earnings stripping}

\section{Aspectos generales}

La evolución de las TCR demuestra que, el earnings stripping (i.e. traslado de beneficios) -propiamente aquello que buscan combatir los estados - es un fenómeno más extenso que la subcapitalización. Por este motivo los países fueron ampliando progresivamente el ámbito de aplicación de las referidas cláusulas para incorporar nuevos supuestos (por ejemplo, los préstamos otorgados por terceros).

Sin embargo, las TCR mantenían el diseño del ratio de endeudamiento sobre el patrimonio. Justamente, diversos autores y organismos internacionales (i.e., OECD) denunciaron la ineficacia de esta estructura. En concreto, señalaban que las TCR permitían que las MNE's manipulen fácilmente el resultado del test; pues bastaba que se efectúen aportes de capital con el fin de evitar que las empresas superen el ratio. En ese sentido, a pesar que las empresas - formalmente - no estaban subcapitalizadas podían aprovechar los beneficios fiscales del earnings stripping.

Precisamente, por este motivo los países diseñaron técnicas alternativas para combatir el problema de la erosión de la base imponible y traslado de beneficios a jurisdicciones con baja o nula imposición, dejando a la subcapitalización como un problema secundario. El primer país en combatir el earnings stripping exclusivamente a través de una ECR fue Alemania, posteriormente, lo imitaron Italia y España.

Estas normas disponen que, una compañía podrá deducir como gasto los intereses hasta un límite determinado por un porcentaje de los ingresos de la empresa (usualmente, se toma como referencia el EBITDA tributario). De esta manera los estados se aseguran que, al menos, una proporción de las utilidades de dicha compañía se encontrará gravada en el país. La aplicación de este método consta de tres pasos35:

1) Determinar apropiadamente el EBITDA de la empresa;

2) Aplicar (multiplicar) el porcentaje establecido en la norma al EBITDA para determinar el monto máximo de interés deducible; y

3) Finalmente, comparar el monto anterior con el interés neto (i.e. gastos por intereses menos ingresos por intereses) de la compañía. El monto que supere dicho límite no será deducible como gasto.

\section{Las earnings stripping rules y la Acción 4 de BEPS}

35 OECD. Limiting Base Erosion Involving Interest Deductions and Other Financial Payments, Action 4 2016 Update: Inclusive Framework on BEPS. París: OECD Publishing, 2017, p. 52. 
Sin perjuicio de que algunos países europeos fueron reemplazando las TCR por ESR; en definitiva, la OECD ha jugado un papel determinante en la promoción de este tipo de cláusula. En definitiva, como se verá en el siguiente apartado, a partir de la recomendación de OECD, el uso de las ESR se ha extendido a otros continentes como Asia (e.g. Japón) y América (e.g. Perú).

El Reporte Final de la Acción 4 de BEPS, publicado en 2015, establece el marco legislativo para combatir la erosión de la base imponible y el traslado de las ganancias ("BEPS o earnings stripping"). Precisamente, en la introducción del documento, se analiza cuál es la mejor técnica para combatir dicho fenómeno, incluyendo las TCR, el enfoque arm's length y las ESR.

En este sentido, la OECD descarta la aplicación de las TCR, y se inclina por las ESR como el mejor método para combatir el problema del earnings stripping. Esto es entendible dado que, este tipo de normas combate directamente el problema de fondo, es decir, el abuso de la deducción de intereses con el fin de erosionar la base imponible.

Por otro lado, la Reporte Final de la Acción 4 de BEPS desarrolla el alcance y estructura de las ESR. En esa línea, partiendo las variables utilizadas para analizar las TCR, la OECD recomienda la aplicación de una ESR con las siguientes características:

a. El límite de intereses deducibles es un porcentaje (entre el 10\% o 30\%) del EBITDA de los ingresos del ejercicio anterior.

b. Coherentemente con el punto anterior, se recomienda una limitación general de los intereses, dejando de ser relevante su origen (i.e., si fue otorgado por una parte vinculada o por un tercero).

c. De igual forma, la consecuencia de la aplicación de la ESR es la prohibición de los intereses que excedan el límite. De hecho, en ninguna parte del documento se considera que la consecuencia sea la recategorización a dividendos.

d. En cuanto a su objeto, la OECD reconoce que el earnings stripping es un fenómeno propio de los grupos empresariales y, particularmente de las MNEs. Por lo mismo, recomienda que los estados apliquen la ESR como mínimo a las empresas que formen parte de un grupo multinacional 36.

En definitiva, se corrobora que las ESR recomendadas por la OECD reúnen las características que se fueron incorporando progresivamente los estados en las TCR y añade una nueva forma de calcular el límite máximo de intereses deducibles. Ciertamente, esto es una muestra que, los estados han dejado de combatir -

36 Sin perjuicio de ello, la OECD prevé la posibilidad que los países amplíen el ámbito de aplicación de las ESR a las empresas que formen parte de un grupo nacional, e incluso a las empresas que no formen parte de ningún grupo.

Íbid., p. 39. 
propiamente - la subcapitalización y han ido enfocando sus esfuerzos para combatir el earnings stripping que, al ser un fenómeno más amplio, requiere de normas con un mayor alcance.

Ahora bien, reconociendo que la norma recomendada tiene un alcance sumamente amplio; la OECD también plantea una serie de medidas adicionales para excluir del ámbito de aplicación a aquellas empresas que no representan un riesgo real de earnings stripping.

a. En primer lugar, recomienda incorporar un umbral mínimo de intereses netos cuya deducción se encuentre permitida, que excluya a las entidades que representen un riesgo mínimo de profit shifting.

b. En segundo lugar, recomienda la incorporación del arrastre de: (a) los intereses que hubieran superado el límite establecido por el ratio; y, (b) arrastre de la capacidad de intereses no utilizada (i.e. sí en un periodo los intereses deducidos son menores al límite determinado por la ratio, la diferencia podrá utilizarse en los ejercicios siguientes).

c. Asimismo, se recomienda establecer disposiciones específicas para el sector financiero.

d. Por último, la OECD recomienda incorporar un ratio especial, subsidiario, para los grupos empresariales, mediante el cual se permita la deducción de intereses mayor al límite general.

Bajo este escenario, la Acción 4 del Plan BEPS realiza recomendaciones para atacar la elusión de grupos multinacionales, la cuales se esquematizan en:

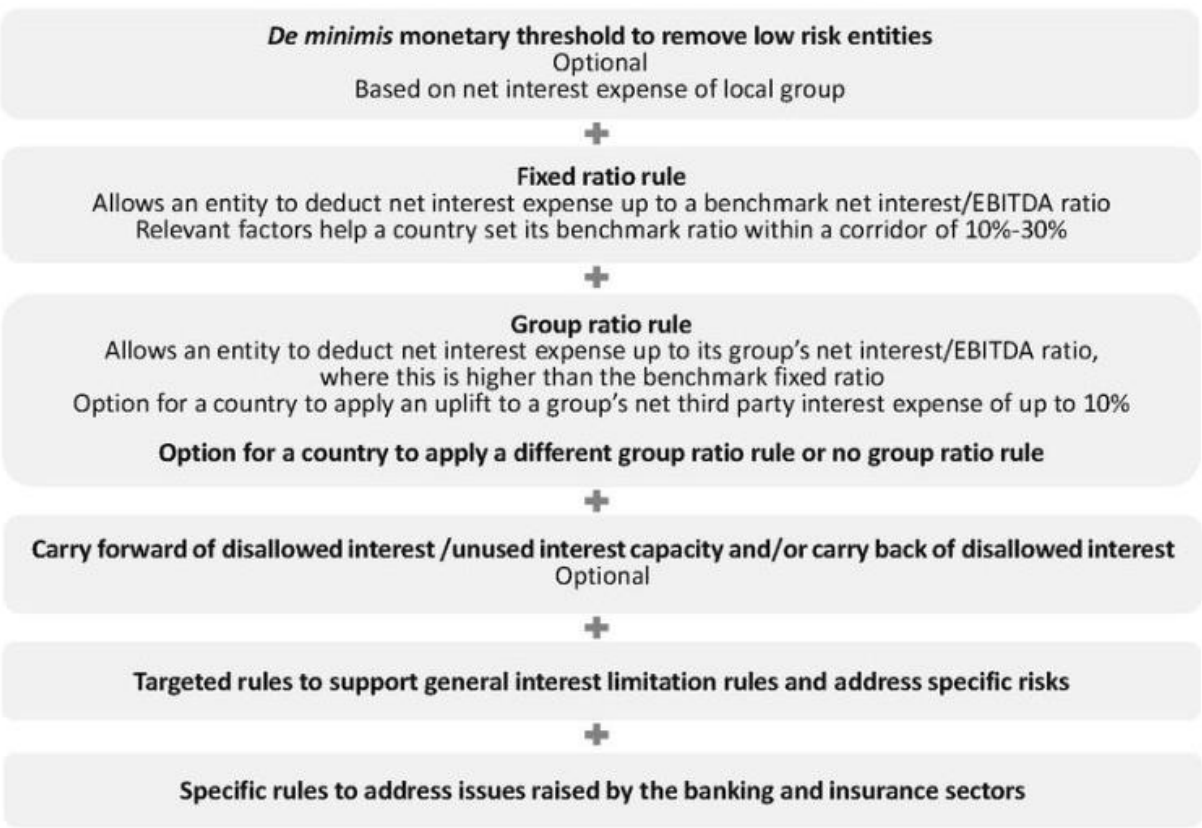

iv. Mecanismos para combatir la subcapitalización y el earnings stripping en la legislación comparada 
En el apartado anterior se ha descrito, conceptualmente, los principales mecanismos para combatir la subcapitalización. Asimismo, se pudo apreciar cómo los estados fueron ampliando las TCR, incorporando características que no eran propias de las cláusulas de este tipo. Este periodo intermedio derivó en el diseño nuevas cláusulas para combatir un fenómeno más amplio; esto es, el earnings stripping. Así pues, con el propósito de aterrizar los conceptos descritos previamente, en el presente apartado se describirán las TCR o ESR de cinco países.

\section{a) La "Zinsschranke" alemana.}

Alemania fue el primer país en reemplazar su TCR por una ESR, a partir de las modificaciones introducidas en el 200837.

En virtud de la "Zinsschranke" son deducibles los intereses netos (i.e., diferencia entre el interés pagado menos el interés percibido como ganancia) que no superen el 30\% del EBITDA38 del ejercicio anterior de una compañía. Su aplicación alcanza a los préstamos otorgados por empresas vinculadas y por terceros son vinculados39. De igual forma, se aplica a las inversiones extranjeras y nacionales, con el propósito de cumplir con los precedentes de la Corte Europea de Justicia40.

La norma alemana incorpora varias exclusiones con el objetivo de que las pequeñas y medianas empresas no se vean afectadas con la aplicación de la norma. Así pues, establece un umbral mínimo de intereses deducibles de 3 millones, el cual es deducible incluso si el endeudamiento total de la empresa supera el 30\% del EBITDA. Asimismo, la norma no aplica si es que la sociedad no forma parte de

37 Según los autores, la modificación se debió a dos motivos: (a) por un lado, aumentar el (bajo) coeficiente de patrimonio en el financiamiento de las compañías alemanas, y (b) por otro lado combatir la erosión de la base imponible de compañías alemanas a través del traslado de sus ingresos a países del extranjero (i.e. earnings stripping).

RUF, Martin y SCHINDLER, Dirk. "Debt Shifting and Thin Capitalization Rules - German Experience and Alternative Approaches". En Nordic Tax Journal, núm. 1, 2015, p. 23.

38 El EBITDA se calcula sobre los ingresos grabados (i.e. considerando la aplicación de pérdidas arrastrables) más los intereses netos y más la depreciación.

39 Los autores agregan que, las autoridades fiscales alemanas habrían estado preocupadas respecto al uso del crédito otorgado por terceros no vinculados (crédito externo) como parte del esquema del earnings stripping. Por este motivo, se amplió el ámbito de aplicación, de tal manera que abarque los préstamos otorgados por entidades vinculadas y por terceros no vinculados.

RUF, Martin y SCHINDLER, Dirk. "Debt Shifting and Thin Capitalization Rules - German Experience and Alternative Approaches". En Nordic Tax Journal, núm. 1, 2015, p. 23.

40 El autor desarrolla el caso Lankhorst-Hohorst (C-324/00). La Corte Europea de Justicia determinó que las TCR Alemanas violaban la libertad de establecimiento prevista en el artículo 43 del Treaty Establishing the European Community (EC Treaty). El autor, citando a los profesores VON BROCHE, K. y GARCÍA PEREZ, E., señaló lo siguiente: “In that case a Dutch firm lent $€ 1.5$ million to its German subsidiary, Lankhorst-Hohorst $\mathrm{GmbH}$, in which it owned 100 percent of the shares. As part of the loan, the parent wrote a letter of support that waived the right to repayment in the event third-party creditors made claims against the German subsidiary. This loan enabled the subsidiary to reduce its bank borrowing and its interest expense. German tax authorities denied the interest deduction and deemed the interest payments to the Dutch owner a dividend distribution, reasoning that a third party would not have made a loan under the same conditions, given the firm's high level of indebtedness and the parent's agreement to waive repayment in favor of other creditors"

WEBBER, Stuart. "Thin Capitalization and Interest Deduction Regulations". Discussion Paper No. 8. Copenhague, Dinamarca: Copenhagen Group on International Taxation - CORIT, p. 692. 
un grupo empresarial. Por último, se prevé que las normas no serán aplicables si la sociedad alemana demuestra que su ratio de endeudamiento es igual o menor que el promedio del ratio de endeudamiento del grupo.

Conforme se puede apreciar, la norma alemana (anterior a las recomendaciones de la Acción 4 de BEPS) comparte la mayoría de las características recomendadas por la OECD.

\section{b) El caso español}

El año 2012, España introdujo grandes modificaciones a sus normas tributarias con la finalidad de reducir el déficit público y la corrección de los desequilibrios de su economía. Al respecto, nos parece importante citar a la Exposición de Motivos del Real Decreto-ley 12/2012 (RDL), norma que introdujo las mencionadas modificaciones:

Se considera una, "prioridad conseguir un incremento de los ingresos fiscales procedentes del Impuesto sobre Sociedades41, esfuerzo recaudatorio que se recaba, fundamentalmente, de las grandes empresas, poseedoras de la capacidad contributiva necesaria para coadyuvar al sostenimiento de las finanzas públicas, a través de la figura del mencionado tributo42".

Así pues, la modificación del 2012 derogó la TCR43 e introdujo una ESR bastante similar a la norma alemana previamente detallada. Precisamente, la norma establece un límite de intereses deducible equivalente al 30\% del beneficio operativo del ejercicio (concepto similar al EBITDA). En cuanto a su alcance, la ESR se aplica a préstamos provenientes de entidades vinculadas y no vinculadas.

En cuanto a sus limitaciones, también incorpora un umbral mínimo de deducción de 1 millón de euros. Asimismo - de manera similar a la norma alemana incorpora: (a) un arrastre de los intereses netos no deducibles hasta por los 18 ejercicios siguientes; y (b) si los gastos financieros netos no alcanzan el límite de endeudamiento, la diferencia podrá consumirse (añadirse) en los cinco años inmediatos y sucesivos44.

\section{c) El caso mexicano}

De acuerdo con el artículo 28 (parágrafo XXVII) de la Ley del Impuesto sobre la Renta Mexicana los intereses que excedan del tripe de su capital contable y, que

41 Impuesto equivalente al IR de tercera categoría.

42 SUÁREZ DE CENTI MARTÍNEZ, Luis y VIANA BARRAL, Víctor. "La limitación en la deducibilidad de gastos financieros en el impuesto sobre sociedades: análisis normativo y comentario crítico". En Actualidad Jurídica Uría Menéndez, núm. 33, 2012, p. 19.

43 Que había quedado bastante mermado, pues debido a las modificaciones introducidas debido al precedente Lankhorst-Hohorst, excluyó de su aplicación a las deudas con entidades residentes de la Unión Europea.

44 SUÁREZ DE CENTI MARTÍNEZ, Luis y VIANA BARRAL, Víctor. "La limitación en la deducibilidad de gastos financieros en el impuesto sobre sociedades: análisis normativo y comentario crítico". En Actualidad Jurídica Uría Menéndez, núm. 33, 2012, p. 23. 
provengan de deudas contraídas con partes relacionadas residentes en el extranjero no serán deducibles (esto es, ratio de 3/1 entre el importe del endeudamiento y el capital contable).

Si bien no establece, en estricto, una prueba en contrario, la norma permite que las empresas puedan ampliar el límite de deducción “(...) en los casos en que los contribuyentes comprueben que la actividad que realizan requiere en sí misma de mayor apalancamiento". Para este efecto, se deberá obtener una autorización en los términos previstos en el Código Fiscal45.

Conforme se puede apreciar, la norma mexicana es una TCR. Asimismo, mantiene la mayoría de las características propias de las cláusulas de este tipo. En efecto, su ámbito es restringido únicamente a los créditos obtenidos de partes relacionadas y, que estén localizados en el extranjero. Sin embargo, se aleja de las características de este tipo de normas, en tanto que el efecto de su aplicación es el impedimento de la deducción de los intereses, más no la recalificación de los intereses como dividendos, lo cual se condice con la naturaleza y fundamento de la TCR46.

\section{d) El caso chileno}

De acuerdo con el artículo 41-F de la Ley sobre el Impuesto a la Renta de Chile los intereses47 en beneficio directo o indirecto de otras empresas relacionadas en el exterior que superen el límite de endeudamiento equivalente a tres veces el patrimonio al final del ejercicio (i.e., ratio de 3/1 entre el importe del endeudamiento anual y el patrimonio) se gravarán con un impuesto único (I.U.) de tasa de $35 \%$. Sin perjuicio de ello, el I.U. podrá ser deducido como gasto48.

La norma no se aplicará cuando el deudor sea una entidad cuya actividad haya sido calificada como de carácter financiero por el Ministerio de Hacienda. Sin perjuicio de lo anterior, su endeudamiento con entidades relacionadas e independientes no podrá, durante el año comercial, ser superior al 120\% del total de los créditos otorgados o de los bienes entregados en arrendamiento financiero (como parte de sus actividades); así pues, en caso de que no se corrija el exceso en el plazo de 90 días, se aplicará la norma de subcapitalización.

Conforme se puede apreciar, la norma chilena no es propiamente ni una ESR ni una TCR, en los términos descritos en el presente trabajo. En efecto, la consecuencia de su aplicación no es ni la recalificación de los intereses en

45 Cámara de Diputados del H. Congreso de la Unión (2013). Ley del Impuesto Sobre la Renta, artículo 28. Consulta realizada el 26 de julio de 2020. Disponible en: http://www.diputados.gob.mx/LeyesBiblio/pdf/LISR_091219.pdf

46 CHIRINOS SOTA, Carlos. "Redescubriendo realidades y formas. La norma Anti Subcapitalización como cláusula Antielusiva Especial". En Derecho \& Sociedad, núm. 31, pp. 295304.

47 El concepto de interés incluye a las comisiones, remuneraciones por servicios y gastos financieros y cualquier otro recargo convencional, incluyendo los que correspondan a reembolsos, recargos de gastos incurridos por el acreedor.

48 Gobierno de la República de Chile (1974). Ley sobre Impuesto a la Renta. Consulta realizada el 27 de julio de 2020. Disponible en: https:/ / nuevo.leychile.cl/navegar?idNorma $=6368$ 
dividendos, ni el impedimento de su deducción como gasto; sino, la aplicación de un impuesto adicional.

Sin perjuicio de ello, su estructura y alcance comparte la mayoría de las características de una TCR. Así pues, el límite de intereses se establece con base en un ratio de endeudamiento sobre patrimonio; y, su alcance se restringe a los intereses originados en préstamos otorgados por partes vinculadas que estén domiciliadas en el exterior.

Finalmente, es importante destacar que, la norma chilena incorpora las recomendaciones de la OECD respecto a la necesidad de establecer disposiciones específicas para las empresas del sector financiero, debido a las particularidades de esta actividad.

\section{v. Diferencias entre la subcapitalización y el earnings stripping}

En los apartados anteriores se analizó, en primer lugar, la diferencia entre los fenómenos de la subcapitalización y del earnings stripping. Posteriormente, se abarcó cuáles han sido las técnicas legislativas utilizadas por los estados para combatir ambos fenómenos. Precisamente, con ello, se pudo verificar que las características de estas técnicas responden al tipo de fenómeno que combaten.

En ese sentido, a modo de conclusión de la primera parte del trabajo, se destacarán las diferencias de cada uno de estos fenómenos y, mostrará cómo estas diferencias repercuten en el diseño de las normas.

La primera diferencia es conceptual. Mientras que la subcapitalización, como fenómeno societario, es la situación de desproporción (prevalencia) del endeudamiento sobre el patrimonio en el financiamiento de una compañía; el earnings stripping es, específicamente una modalidad de planificación fiscal propia de las MNEs, mediante la cual se erosiona la base fiscal de una compañía y se traslada los ingresos gravables a otras jurisdicciones.

Sin embargo, en el ámbito tributario, esta diferencia pierde claridad. Precisamente, los autores definen a la subcapitalización (tributaria) como la situación de desproporción (prevalencia) entre el endeudamiento sobre el patrimonio ocasionada por la decisión deliberada del accionista con el objetivo de erosionar la base imponible y, así obtener una ventaja tributaria.

Así, sin perjuicio de la definición tributaria de subcapitalización, el earnigns stripping sigue siendo un fenómeno más amplio. El cual puede aparecer, incluso, cuando la compañía no se encuentre subcapitalizada. Véase, el aprovechamiento de esta práctica puede realizarse a través del otorgamiento de préstamos (en países 
con alta imposición), e incluso, a través de aportes de capital (en países con baja imposición).

La segunda diferencia es de carácter subjetivo. Cabe recordar que, la subcapitalización es la desproporción entre el endeudamiento de una sociedad y su capital social, ocasionada por la decisión deliberada de los accionistas de financiar a la empresa mediante préstamos, en lugar de aportes de capital. Siendo ello así, los agentes relevantes en la subcapitalización son: (a) el accionista (el cual, regularmente es la sociedad matriz); y, (b) la empresa subcapitalizada (la cual, regularmente, es la subsidiaria o filial). Nótese que, la subcapitalización se puede presentar, aún cuando el accionistas y la empresa estén establecidas en el mismo territorio. De igual forma, los terceros (esto es, sociedades ajenas al grupo empresarial) no tienen participación dentro de este fenómeno49.

En cambio, el earnings stripping, es un fenómeno propio de las MNEs (grupos multinacionales); por lo que, en estricto, resulta necesario que el accionista y la empresa financiada estén establecidas en distintas jurisdicciones. De igual forma, los terceros, sí juegan un papel relevante en el earnings stripping. Conforme hemos detallado, las MNEs puede ejecutar esta práctica a través de financiamientos obtenidos de terceros en jurisdicciones con una alta imposición. En suma, los agentes relevantes en el earnings stripping son: (a) el accionista (parte de un grupo multinacional), (b) el prestatario (matriz u otra entidad parte de un grupo multinacional); (c) eventualmente, terceros prestatarios (por ejemplo, bancos).

Relacionado con el punto anterior, la tercera diferencia se verifica en el tipo de financiamiento relevante para cada fenómeno. Precisamente, en la medida que los terceros son completamente ajenos al fenómeno de la subcapitalización; por naturaleza, la TCR solo restringirá la deducción de los intereses originados en préstamos de partes vinculadas. En cambio, dado que el earnigns stripping puede generarse con el financiamiento de terceros; las ESR establecen una limitación general a la deducción de gastos financieros (sin distinguir si se originan en préstamos de terceros o de partes vinculadas).

Estas diferencias en cada uno de los conceptos han quedado plasmadas, a su vez, en la estructura, alcance y efectos de las técnicas legislativas empleadas por los estados para combatir ambos fenómenos; conforme se puede apreciar en el siguiente cuadro:

\section{\begin{tabular}{|l|l|l|} 
Característica & Thin capitalization rule & Earning stripping rule
\end{tabular}}

49 Conforme fue detallado previamente, es posible que los accionistas encubran sus financiamientos, utilizando a un tercero como intermediario (estructuras back to back). No obstante, en esta situación, el tercero continúa siendo ajeno a la subcapitalización, puesto que actúa como un simple intermediario; siendo que el accionista continúa siendo el verdadero prestatario. 
¿Regla de subcapitalización o earnings stripping rule?: Análisis evolutivo y comparativo de las normas que limitan la deducción de gastos financieros y su enfoque en el Perú

\begin{tabular}{|c|c|c|}
\hline $\begin{array}{l}\text { Estructura de la } \\
\text { cláusula }\end{array}$ & $\begin{array}{l}\text { Ratio de endeudamiento sobre } \\
\text { patrimonio (debt to equity). }\end{array}$ & $\begin{array}{l}\text { El límite de la deducción de } \\
\text { intereses es un porcentaje de } \\
\text { los ingresos, generalmente se } \\
\text { toma como referencia el } \\
\text { EBITDA. }\end{array}$ \\
\hline Créditos relevantes & $\begin{array}{l}\text { Otorgados por partes vinculadas. } \\
\text { En algunas jurisdicciones, se } \\
\text { establece el requisito de que el } \\
\text { prestamista cuente con una } \\
\text { participación mínima en el } \\
\text { patrimonio de la prestataria. }\end{array}$ & $\begin{array}{l}\text { Otorgados por partes } \\
\text { vinculadas y por terceros. }\end{array}$ \\
\hline \multirow{2}{*}{$\begin{array}{l}\text { Procedencia de las } \\
\text { inversiones }\end{array}$} & $\begin{array}{l}\text { Por naturaleza, no debería ser } \\
\text { relevante el origen. No obstante, era } \\
\text { usual que la aplicación de la TCR se } \\
\text { restringiera a las inversiones } \\
\text { (financiamientos) extranjeras50. }\end{array}$ & $\begin{array}{l}\text { Por naturaleza, debería } \\
\text { restringirse a las inversiones } \\
\text { (financiamientos) extranjeros } \\
\text { (pues es el ES es un fenómeno } \\
\text { inherente a las MNEs51) }\end{array}$ \\
\hline & \multicolumn{2}{|c|}{$\begin{array}{l}\text { Sin perjuicio de lo anterior, en la actualidad, no es usual que las TCR o } \\
\text { ESR - especialmente de los países europeos - discriminen la } \\
\text { procedencia de las inversiones debido a los precedentes de la Corte } \\
\text { Europea de Justicia y el principio de libertad de establecimiento dentro } \\
\text { de la Unión Europea. }\end{array}$} \\
\hline Efecto & $\begin{array}{l}\text { Recategorización de los intereses } \\
\text { como dividendos, gravándose con } \\
\text { el impuesto correspondiente. }\end{array}$ & $\begin{array}{l}\text { Limitación general a la } \\
\text { deducción de intereses (gastos } \\
\text { financieros). }\end{array}$ \\
\hline
\end{tabular}

Fuente: Elaboración propia

No obstante, si bien estas diferencias son, conceptualmente, claras, los diferentes estados no han plasmado con exactitud tales diferencias.

En efecto, conforme se ha podido apreciar, inicialmente, las TCR fueron diseñadas siguiendo las características que hemos detallado (e.g., la norma alemana y española en su versión original). Sin embargo, progresivamente, fueron incorporando elementos que, propiamente, no responden a su naturaleza.

Por ejemplo, la TCR mexicana vigente no sanciona con la recalificación de los intereses a dividendos, sino con su no deducibilidad. De igual, en otros países se amplió el ámbito de aplicación a préstamos otorgados por partes vinculadas y no vinculadas.

Desde nuestro punto de vista, le evolución responde a que los Estados fueron progresivamente girando su objeto de preocupación, que originalmente, era la subcapitalización a un fenómeno más amplio, como el earnings stripping.

50 Esto es una muestra que, para efectos tributarios, la subcapitalización solo genera un perjuicio del fisco cuando intervienen empresas de distintas jurisdicciones.

51 Quienes aprovechan: (i) la facilidad para trasladar sus actividades y activos financieros y, (ii) la diferencia de las tasas del IR de los distintos países. 
El paso final de esta evolución es el reemplazo de las TCR por la ESR, que resultarían ser más eficaces para combatir la erosión de la base imponible y el traslado de beneficios a otras jurisdicciones.

\section{Técnicas legislativas para combatir la subcapitalización en el Perú}

El Perú cuenta con un norma de subcapitalización desde hace casi dos décadas; la misma que había permanecido, prácticamente, invariable hasta el 2018, año en el que el gobierno modificó sustancialmente la referida norma. Precisamente, en el presente apartado se analizará la evolución de la norma de subcapitalización peruana, tomando como referencia, el marco teórico expuesto en la primera parte del presente trabajo.

\section{i. Historia de las normas de subcapitalización en el Perú}

\section{a) Primera norma de subcapitalización}

La primera norma de subcapitalización data del año 2001. Específicamente, mediante el artículo 6 de la Ley $\mathrm{N}^{\circ} 27356$, vigente a partir del 01 de enero de 2001, se incorporó por primera vez una norma de subcapitalización al inciso a) del artículo 37 de la Ley del Impuesto a la Renta (IR), la cual establecía que:

"Serán deducibles los intereses provenientes de endeudamientos de contribuyentes con sujetos o empresas vinculadas cuando dicho endeudamiento no exceda del resultado de aplicar el coeficiente que se determine mediante decreto supremo sobre el patrimonio del contribuyente; los intereses que se obtengan por el exceso de endeudamiento que resulte de la aplicación del coeficiente no serán deducibles" (el énfasis es nuestro).

Pues bien, el inciso a) del artículo 21 del Reglamento de la Ley del IR estableció que el límite de deducción de intereses se hallará aplicando un coeficiente de tres (03) sobre el patrimonio neto de una compañía al cierre del ejercicio anterior (ratio de 3/1). De esta manera, los intereses que se obtengan por el exceso de endeudamiento no son deducibles a efectos de la determinación del IR.

En sus 18 años de vigencia, la única modificación que sufrió la referida norma fue introducida mediante el artículo el artículo 25 del Decreto Legislativo $N^{\circ} 945$ al reemplazar la referencia de "sujetos o empresas vinculadas" por "partes vinculadas". Como se podrá apreciar, se trata de una modificación terminológica, que no altera la estructura de la norma.

Bajo el marco teórico expuesto en este trabajo, la norma de subcapitalización peruana se trata, en efecto, de una norma de subcapitalización del tipo "objetivo", más precisamente una TCR. Justamente, consideramos que se trata de una norma de subcapitalización porque el ratio de endeudamiento se calcula tomando como referencia una comparación entre el endeudamiento y el patrimonio de una sociedad. 
Esto último se condice con el alcance de la norma peruana, la cuál era aplicable únicamente a los endeudamientos de contribuyentes con empresas vinculadas, lo que es propio de una verdadera TCR. Por el contrario, los efectos de la aplicación de la norma sí se alejan de las características propias de una TCR. Así pues, el efecto previsto fue el impedimento de la deducción como gasto de los intereses que excedieran el límite, más no su recalificación como dividendos.

De igual forma, es de notar que, la norma tampoco preveía la posibilidad de que el contribuyente pruebe que, a pesar de superar el límite, en realidad no se encuentra subcapitalizada (esto es, probar que el endeudamiento cumple con el principio de plena competencia o arm's length).

Por otro lado, conforme se puede apreciar, la estructura de la norma sigue la estructura de las TCR que introdujeron, inicialmente, los países europeos, y, es bastante similar a la norma mexicana (aunque sin las excepciones y disposiciones específicas.

Esta semejanza es lógica, teniendo en consideración los siguientes puntos: (a) las primeras TCR empezaron a introducirse desde finales de los años noventa hasta mediados de las primera década del presente siglo; y, (b) hasta el 2008, todos los países, a excepción del Reino Unido, utilizaban TCR para combatir la subcapitalización. Siendo ello así, es lógico que la norma comparta la estructura de las normas europeas.

Por último, otro aspecto que aleja la norma peruana de la de los países europeos es que su aplicación no está dirigida exclusivamente a los grupos empresariales. Como señala, Katarzyna DUNIN, en el Perú no existe una regla de consolidación grupal de resultados financieros52. Por tanto, no sería eficiente estructurar la cláusula de esta manera; por este motivo, la norma simplemente hace referencia a "partes vinculadas".

\section{b) Nueva norma: ¿Dejaremos de tener una norma de subcapitalización en 2021?}

Con la publicación de Decreto Legislativo 1424, se introdujeron dos regímenes: (a) uno transitorio aplicable en los años 2019 y 2020 y, (b) uno definitivo, que aplicará, salvo cambio legislativo, desde el 1 de enero de 2021.

El régimen transitorio extendió la aplicación del límite para la deducción de intereses, tanto para partes vinculadas como independientes, que decidan realizar operaciones de financiamiento a partir del 01 de enero de 2019.

Así pues, con la reciente modificatoria a la regla de subcapitalización, se establece que sólo serán deducibles los intereses provenientes de endeudamientos, cuando 
dichos endeudamientos no excedan del resultado de aplicar el coeficiente 3 sobre el patrimonio neto del contribuyente al cierre del ejercicio anterior; los intereses que se obtengan por el exceso de endeudamiento que resulte de la aplicación del coeficiente no serán deducibles.

La nueva regulación excluye de la aplicación del límite a los contribuyentes que pertenezcan a empresas del sistema financiero y de seguros; contribuyentes cuyos ingresos netos en el ejercicio sean iguales o menores a 2,500 UIT; contribuyentes cuyo endeudamiento se destinen para el desarrollo de proyectos de infraestructura pública, servicios públicos, servicios vinculados a estos, investigación aplicada y/o innovación tecnológica, bajo la modalidad de Proyectos en Activos; y contribuyentes cuyo endeudamiento provenga de la emisión de valores mobiliarios nominativos representativos de deuda que cumplan con las condiciones que establece el Decreto Legislativo en mención.

Respecto a este régimen, debemos mencionar que, en estricto continúa siendo una norma de subcapitalización, en la medida que el ratio de endeudamiento se calcula tomando como referencia el patrimonio de una sociedad.

Ciertamente, al haber tomado como referencia las recomendaciones de la OECD, la modificación ha introducido elementos que son más propios de una ESR53. Precisamente el gran cambio de la norma radica en la ampliación del ámbito de aplicación objetivo de la TCR a endeudamientos de cualquier tipo (sin distinguir si fueron otorgados por partes vinculadas o por terceros). Justamente, los países europeos ampliaron sus TCR, esencialmente, por dos motivos: (a) las MNEs erosionan la base imponible de sus filiales, incluso, a través del endeudamiento proveniente de terceros (por ejemplo, al endeudarse obteniendo préstamos en países con una alta imposición); y, (b) las MNEs utilizaban operaciones del tipo back to back, en las que utilizaban como intermediario a una sociedad no vinculada y, con ello burlaban las normas de subcapitalización. Es de notar que, el primer motivo es completamente ajeno a la subcapitalización.

$\mathrm{Al}$ respecto, nos preguntamos si el Estado peruano ha detectado prácticas de este tipo - que conlleven un real perjuicio para el país - o se dejó llevar por consideraciones ajenas (Acción 4 de BEPS).

A partir de 2021, el régimen cambiará drásticamente, tanto así, que, en estricto, el Perú dejará de tener una norma de subcapitalización y se unirá al resto de países que las han reemplazado por las earnings stripping rules.

La norma aplicable desde el 1 de enero de 2021 incorpora como regla general el límite para la deducción de gastos por intereses con una ratio fija equivalente al $30 \%$ del EBITDA54 del ejercicio anterior, es decir, los intereses netos que excedan

53 Esto es lógico, dado que la Acción 4 de BEPS establece el marco recomendado para combatir la erosión de la base imponible y el traslado de ganancias, más no contra la subcapitalización.

54 Se debe entender por EBITDA a la renta neta luego de efectuada la compensación de pérdidas más los intereses netos, depreciación y amortización. 
el 30\% del EBITDA del ejercicio anterior serán sancionados a efectos del cálculo del IR55.

Siguiendo la línea de este trabajo, consideramos que la nueva normativa (que entrará en vigencia en 2021) introduce una ESR, pues no combate la subcapitalización (desproporción entre el patrimonio y el endeudamiento (externo) de una sociedad); si no, de manera general, el earnings stripping (erosión de la base imponible y el traslado de beneficios a otras jurisdicciones); justamente, a través de una limitación general a la deducción de intereses (sin importa si hay o no subcapitalización) 56 .

$\mathrm{Al}$ respecto, cabe mencionar que, los países que han adoptado este tipo de norma justifican su adopción por dos motivos: (a) porque quieren combatir de manera efectiva el fenómeno del earnings stripping o BEPS, y/o (b) porque se ha demostrado que el ratio de endeudamiento debt/equity es ineficiente.

Sobre lo primero, cabe mencionar que, como señalamos previamente, el earnings stripping es inherente a las compañías multinacionales (MNEs). Al respecto, planteamos unas preguntas que, exceden por mucho el alcance del presente trabajo, pero pueden ser materia de un estudio posterior: ¿Cuántas compañías de este tipo hay en el Perú? ¿Justifica este número adoptar una normativa orientada exclusivamente hacia ellas? ¿La legislación tributaria peruana es atractiva para las MNEs a fin de realizar prácticas de este estilo?, ¿El earnings stripping es realmente un problema para el Perú?

En resumen, tanto la primera norma de subcapitalización, incorporada en 2001 y la nueva norma de "subcapitalización" vigente desde el año pasado, han sido el resultado de acoger las tendencias del momento. La primera, sería el resultado de seguir la tendencia de los países europeos de adoptar normas de subcapitalización basadas en un ratio de endeudamiento del tipo debt/equity. La segunda, adopta la tendencia de los países europeos, que finalmente ha sido recogida por la OECD en la Acción 4 del Plan de BEPS de abandonar las viejas TCR y optar por una del tipo earnings stripping.

Ciertamente, seguir las tendencias mundiales no tiene nada de malo, sin embargo, ello debe ir acompañado de un profundo análisis de la realidad de los países y de cuál es su problemática real. Por eso, consideramos que la nueva normativa tiene problemas estructurales y funcionales que analizaremos las siguientes páginas de este trabajo.

55 El Decreto Supremo 338-2018-EF modificó el Reglamento de la Ley del IR e introdujo, entre otras disposiciones el procedimiento para determinar el Interés Deducible Máximo (IDM) a partir de una operación aritmética entre el Monto Máximo de Endeudamiento (MME), el Monto Total de Endeudamiento y el Monto de Interés (MI) que cumpla con el principio de causalidad. Dicha reglamentación entró en vigencia el 01 de enero de 2019.

56 A pesar de esto, la Exposición de Motivos fundamenta la adopción de la nueva norma porque ayudará a combatir con la subcapitalización. 


\section{ii. Problemas estructurales de la nueva norma}

En este punto analizaremos los problemas estructurales de la regla de limitación de intereses establecida por el D.L. 1424 y el Decreto Supremo 338-2018-EF. Para ello, tendremos en consideración las Acción 4 del Plan BEPS y las características propias nuestro ordenamiento jurídico tributario.

\section{a) Finalidad Antielusiva}

La principal finalidad para implementar una regla que limita la deducibilidad de gastos financieros es combatir la elusión tributaria. Así, como se explicó en los apartados anteriores, la forma elusiva en este caso se configura erosionando la base tributaria de países de alta imposición a través de deducción de intereses y transfiriendo el rendimiento a través de intereses hacia un país de menor imposición.

Siendo este el principal objetivo de dicha norma y considerando que existe una norma predecesora en nuestro ordenamiento, resulta coherente que el legislador tributario haya identificado dos cosas: (i) que en el Perú es frecuente la utilización de este mecanismo para erosionar la base tributaria y (ii) que la actual norma de limitación de intereses fracasó en su fin antielusivo y los motivos de tal fracaso57.

Un primer indicio de la exigua investigación que se ha hecho para implementar esta medida se observa cuando la Exposición de Motivos del D.L. 1424 señala que “[e]n efecto, a fin de aprovechar las ventajas tributarias y sin tener en cuenta razones empresariales, los contribuyentes pueden optar por realizar aportes de capital encubiertos mediante la asignación de recursos a una empresa bajo la modalidad de un préstamo".

Del texto citado, se desprende que el legislador no asegura que los contribuyentes del fisco peruano utilizan un esquema elusivo basado en la deducción de intereses, sino que simplemente existe la posibilidad abstracta de que lo hagan. Y remarcamos "posibilidad abstracta" porque tampoco se sustenta que los contribuyentes peruanos están en las condiciones para poder realizar estos esquemas (cuyo benchmark está pensado en las MNEs).

Por otro lado, la Exposición de Motivos señala que:

“A pesar de la incorporación de dicho límite en la LIR, en los últimos años los gastos financieros de los contribuyentes se han incrementado significativamente. En efecto, se ha observado que en el 2017 estos gastos aumentaron en un 32,1\% respecto del 2012, porcentaje elevado en comparación al incremento al incremento del número de contribuyentes en dicho periodo el cual ascendió a 8.6\%. Asimismo, en el 2014 como

57 Este punto es sumamente importante porque la Acción 4 de BEPS propone (a su entender) el mejor mecanismo para evitar la erosión de la base tributaria a través de la deducción de intereses, pero reconoce que existen otras fórmulas que podrían funcionar de acuerdo con las características propias de cada país. 
consecuencia de la reducción de la base imponible por la deducción de gastos financieros disminuyó el IR determinado".

$\mathrm{Al}$ respecto, debemos señalar que no se demuestra que el aumento de los gastos financieros corresponde al uso de mecanismos elusivos, ello sobre todo considerando que el aumento de gastos por intereses puede estar relacionado a otros factores de mercado o de inversión, ajenos a fines elusivos.

Asimismo, no se sustenta que los gastos financieros están relacionados préstamos provenientes de países de baja o nula imposición, para que de manera conjunta se refleje en una mayor "eficiencia tributaria" en favor de la compañía multinacional que realiza este tipo de mecanismos. Por otro lado, se debe de considerar que los préstamos pueden venir de contribuyentes localizados en el país (operaciones dentro de una misma jurisdicción) cuyo rendimiento por intereses estará gravado por la misma tasa del impuesto a la renta le correspondería a la empresa cuya base impositiva fuera presuntamente erosionada.

Cabe resaltar que, una reducción del pago del impuesto a la renta por la deducción de gastos financieros no demuestra per se que se estén utilizando mecanismos elusivos. En ese sentido, no entendemos por qué se ha hecho esa precisión, a menos que esta parte de la Exposición de Motivos revele que la verdadera intención de la norma tiene cariz recaudatorio más que anti-elusivo.

Por otro lado, debemos resaltar que otro de los argumento en los que se basa la Exposición de Motivos para sustentar la reforma a la regla del límite de deducción de intereses es la evidencia internacional que demostraría que los contribuyentes pueden adoptar esquemas complejos bajo los cuales no es posible verificar si se están realizando operaciones entre partes vinculadas tales como aquellos en los que se utiliza un tercero como intermediario, lo que se denomina back to back.

Sobre este punto, no solo llama la atención que la Ley del Impuesto a la Renta ya prevé un tratamiento específico a este tipo de operaciones "castigando" con una tasa de retención del 30\% en el inciso j) del artículo 5658 del mencionado cuerpo normativo, sino que, adicionalmente y de manera reiterativa, se omite demostrar que los contribuyentes del fisco peruano efectivamente realizan esta operación como mecanismo elusivo (o incluso si están en las condiciones económicas para poder realizarlas).

Cabe señalar que en tal referencia se ampara la Acción 4 del Plan BEPS para sugerir que los límites a la deducción de intereses deben ser aplicados también a partes independientes, debido a que estas al ser MNEs estarían estructuradas bajo figuras societarias complejas, hecho que tampoco ha sido sustentado en la Exposición de Motivos y que, claramente, podría diferir mucho de la realidad nacional.

58 Esta norma señala que se entiende que existe una operación de crédito en donde la intervención del acreedor ha tenido como propósito encubrir una operación entre empresas vinculadas, cuando el deudor domiciliado en el país no pueda demostrar que la estructura o relación jurídica, adoptada con su acreedor coincide con el hecho económico que las partes pretenden realizar. 
De todo lo expuesto, consideramos que el aumento de gastos financieros y la subsecuente disminución en la determinación del IR no resultan por sí solos argumentos válidos para establecer una nueva regla a la limitación a la deducción de intereses, pues dichos fenómenos no sustentan que se estén utilizando dichas operaciones como mecanismos elusivos.

Por otro lado, la Exposición de Motivos señala que "Existen indicios de que estas formas de endeudamiento se vienen realizando en nuestro país, ya que empresas sin patrimonio o con bajas utilidades reciben préstamos desproporcionados".

En este punto, debemos reiterar que, como se señaló en apartados anteriores, si bien el fenómeno de la subcapitalización está relacionado con la erosión de la base impositiva a través de la deducción indebida de interés, dicho fenómeno por sí solo no suponen actos elusivos entre los contribuyentes.

Ahora, si bien el texto es claro al señalar que este hecho se trata de un indicio, consideramos que tal indicio no sería suficiente para sustentar un cambio de régimen más riguroso que implique la inclusión de operaciones de financiamiento entre partes vinculadas y el uso del EBIDTA para establecer el límite de interés deducible.

Finalmente, consideramos que una de las deficiencias más notorias de esta norma se encuentra en que no explica las falencias de la anterior regulación en combatir la elusión tributaria y, en ese sentido, tampoco sustenta cuáles serían las ventajas con la nueva regulación (ni la actual ni la aplicable a partir del 2021).

\section{b) Punto de referencia y contexto OECD}

Como se señaló en el apartado anterior, la Exposición de Motivos usa como fuente al Reporte Final de la Acción 4 publicado en el 2015. A su vez, dicho reporte contextualiza su propuesta en las operaciones realizadas por empresas que califican como grupos multinacionales (MNEs) y países miembros de la OECD y del G20.

En relación con los MNEs podemos señalar que, como se explicó de manera previa, se tratan de empresas que por su capacidad productora y la economía globalizada se encuentran operando en más de un país. Hecho que, considerando las variaciones impositivas de cada país, le permite generar eficiencias tributarias indebidas.

Sobre la base de este grupo de empresas, la Acción 4 reconoce que la indebida deducción de intereses resulta ser una práctica frecuente en grupos económicos multinacionales que por su naturaleza se rigen como si fueran un solo ente, esto es, que aun cuando jurídicamente puedan representarse como diferentes personas jurídicas en cada jurisdicción, toman sus decisiones como si fueran uno solo (justamente por pertenecer a un solo grupo comercial). 
Estos grupos pueden realizar operaciones de financiamiento transfronterizo que, alocando de manera oportuna los ingresos y gastos por intereses en sus transacciones de financiamiento, "construyen" operaciones que sobrepasan el límite de la economía de opción.

En este sentido, los principales riesgos que se identifican en la Acción 4 se relacionan a grupos multinacionales que podrían: (i) trasladar sus gastos por intereses a países de alta imposición, (ii) generar gastos de intereses en exceso en el grupo en comparación con los intereses que paga el grupo a terceros no vinculados y (iii) utilizar financiamiento para generar ingresos no gravados.

Ahora bien, siendo este el marco de referencia o benchmark de la Acción 4 del plan BEPS, nos queda preguntarnos si la norma introducida por el D.L. 1424 está dirigido para el mismo grupo contribuyentes en el Perú.

Ante tal interrogante un primer indicio de respuesta la encontramos cuando la Exposición de Motivos indica que “(...) en el 2016 las empresas con ingresos superiores a 2500 Unidades Impositivas Tributarias (UIT) que son principales contribuyentes (PRICOS) tuvieron una elevada concentración de gastos financieros equivalente al 98\% del total de gastos declarados".

Desde nuestro punto de vista, no cabe duda de que las empresas multinacionales a que hace referencia la Acción 4 son compañías que tienen un alto porcentaje de ingresos, sin embargo, como se explicó líneas arriba, tanto el nivel de ingresos como la concentración de gastos financieros no son características que determinen su calificación como MNEs (al menos en los términos de la OECD) que utilizan mecanismos de financiamiento para erosionar la base impositiva.

Como se puede observar, el legislador tributario no ha tomado en consideración que el grupo de referencia en la que se basa la Acción 4 no necesariamente tiene un comparable en nuestro escenario económico o, de tenerlo, tampoco queda demostrado que realicen estos mecanismos elusivos.

Por otro lado, no queda claro que el D.L. 1424 haya tomado en cuenta el contexto en el cual se enmarca las soluciones propuestas por la Acción 4; entendiendo como tal, al grupo de países que dirige las directrices planteadas y al grupo de países al que van dirigido.

$\mathrm{Al}$ respecto, resulta interesante los comentarios que realizó Rob Heferen, Subsecretario del Revenue Group of the Australian Treasury citado por Craig Elliffe, sobre la conveniencia de adoptar el EBITDA como ratio para el límite de deducción de intereses:

"La mayoría de los miembros de la OECD son europeos. El secretario tiene una inclinación fuertemente europea. Así, ellos ven a través de una perspectiva alemana y francesa, porque los ingleses van, pero no les interesa de todas formas, y los norteamericanos no participan. En nuestra situación con altos niveles de imposición corporativa a nivel internacional, 
necesidades altas de inversión en infraestructura, ricos en recursos, y bastante dependientes de capitales extranjeros para potenciar activos potencialmente productivos en activos productivos, tengo la sensación de que estamos en una situación donde necesitamos estar seguros que nuestro criterio de $a r m$ 's- length's se mantenga y sea aceptado por la OECD59".

No cabe duda que, estos comentarios se pueden replicar a nuestro caso, en tanto que el D.L. 1424 toma como referencia la Acción 4 del plan BEPS sin que en la Exposición de Motivas se haga referencia a una evaluación previa de las especiales condiciones de nuestro entorno económico y nuestro ordenamiento jurídico tributario.

Asimismo, llama la atención lo expresado por Suárez de Centi y Viana cuando en sus comentarios al Real Decreto-ley 12/2012 que introduce la regla del EBITDA como ratio para el límite de deducción de intereses en España, señalan que

"Como punto de partida, creemos que una norma dirigida a limitar la deducibilidad de los gastos financieros de las empresas puede suponer un claro freno a la importación de capitales por nuestro país, al reducir la rentabilidad de las inversiones realizadas a través de una sociedad o un establecimiento permanente situados en España en unas circunstancias económicas muy adversas y una situación de difícil acceso al crédito60".

Al respecto, consideramos que este comentario también resultaría aplicable a nuestro caso pues el Perú califica como país importador de capitales desde su apertura económica en la década de los 90’s. Así, creemos que el legislador no ha tomado en consideración que esta nueva regla de limitación intereses tendrá como consecuencia no solo desalentar los comportamientos elusivos (cuya existencia no ha sido corroborada en el fisco peruano) sino, principalmente, la inversión extranjera.

No debemos olvidar que el Proyecto BEPS se ha basado, primordialmente, en la realidad y problemática de los países OCDE y del G20. Así, resulta comprensible que países como Alemania, Suecia o Italia utilicen una regla única de EBITDA en su calidad de países exportadores de capitales y que, en tan sentido, cuentan con reconocidas empresas multinacionales que tienen la opción real de alocar préstamos con fines elusivos61.

59 Traducción propia de: "The majority of the OECD members are European. The secretariat is heavily European based. So they look at it through a German and French perspective, because the British come in and go out and ignore it anyway, and the Americans do not participate. I our situation with relatively high corporate rates internationally, high infrastructure investment needs, resource rich, and pretty reliant on foreign capital to turn potentially productive assets into productive assets, we felt we were in a situation where we needed to make sure that the arm's- length's test was retained, and the OECD accepted that". ELLIFFE, Craig. "Interest Deductibility: Evaluating the Advantage of Earnings Stripping Regimes in Preventing Thin Capitalisation". En New Zealand Law Review, vol. II, 2017, p. 266.

60 SUÁREZ DE CENTI MARTÍNEZ, Luis y VIANA BARRAL, Víctor. "La limitación en la deducibilidad de gastos financieros en el impuesto sobre sociedades: análisis normativo y comentario crítico". En Actualidad Jurídica Uría Menéndez, núm. 33, 2012, p. 23.

61 Incluso en países con características semejantes pero más conservadores en su política fiscal, tales como Dinamarca y Japón, han optado por una regla doble de limitación de intereses 
¿Regla de subcapitalización o earnings stripping rule?: Análisis evolutivo y comparativo de las normas que limitan la deducción de gastos financieros y su enfoque en el Perú

\section{c) Umbral mínimo y Reglas Anti -fragmentación}

En línea con la recomendación del Reporte Final de la Acción 4 del plan BEPS de establecer un mínimo de contribuyentes exceptuados de la regla de subcapitalización, el D.L. 1424 establece como una excepción a todos los contribuyentes cuyos ingresos netos en el ejercicio gravable sean menores o iguales a 2500 UIT.

En primer lugar, no queda del todo claro cuál ha sido el criterio del Estado peruano para establecer la regla de exclusión con base en los ingresos netos de una empresa. Consideramos que, el umbral mínimo elegido por un país debe ser coherente con el alcance de su TCR o ESR. Así pues, si un país adopta una norma del tipo earnings stripping, el umbral mínimo deberá estar basado en un mínimo de intereses neto deducible, tal como lo ha hecho España y Alemania.

Al respecto, la OECD ha señalado lo siguiente:

“Los países podrán incluir un umbral mínimo (de minimis threshold) a fin de excluir sociedades que representen un riesgo bajo del ámbito de aplicación de la norma (fixed ratio rule). Es recomendable que este umbral esté basado en el total de intereses netos de todas las entidades que conforma el grupo local. Si el país quiere aplicar un umbral basado en los intereses netos de cada entidad por separado, es importante que tales reglas no sean abusadas. Por lo tanto, el país deberá considera la inclusión de normas antifragmentación (...)62".

Pues bien, el Estado peruano debería haber adoptado un umbral mínimo para el régimen transitorio vigente en 2019 y 2020 (basado en los ingresos netos de la Compañía); y, otro para el régimen que entraría en vigencia en 2021, estableciendo un mínimo de intereses deducible.

En segundo lugar, la norma peruana tampoco observa la recomendación de la OECD respecto a la necesidad de establecer reglas "anti-fragmentación" Dichas reglas evitarían que compañías con el único ánimo de alocarse dentro del mínimo de exclusión establecida por la norma, atomicen sus operaciones a través de otras personas jurídicas $\mathrm{y}$, finalmente, logren deducir intereses sin ningún tipo de limitación.

Al respecto, el Reporte Final de la Acción 4 señala que cuando se aplica una regla a nivel de una entidad individual, esto es, cuando no se ha considerado una regla de ratio grupal (como en nuestro caso), el país debe considerar establecer reglas de

considerando el EBIDTA junto a un coeficiente del patrimonio o activos como ratios para tal limitación.

62 Traducción propia de: "Countries may therefore introduce a de minimis threshold to exclude ow risk entities from the scope of the fixed ratio rule and group ratio rule. It is recommended that such threshold should be based on the total net interest expense of all entities in the local group. Where a country wishes to apply a threshold based on the net interest expense of each entity separately, it is important that these rules are not abused. Therefore, a country should consider introducing ant-fragmentation rules".

OECD. Limiting Base Erosion Involving Interest Deductions and Other Financial Payments, Action 4 2016 Update: Inclusive Framework on BEPS. París: OECD Publishing, 2017, p. 39. 
anti-fragmentación para evitar que un grupo evite la aplicación de una regla de limitación de intereses al establecer un número de entidades donde cada una de las cuales cae por debajo del umbral mínimo establecido por la norma.

En efecto, la excepción a la aplicación del límite de deducción de intereses para contribuyentes con ingresos menores a las 2500 UIT podría resultar incluso más nociva considerando la finalidad antielusiva de dicha norma, pues compañías que logren atomizar sus operaciones en diferentes empresas de tal forma que de manera individual no superen los 2500 UIT, podrán deducir la totalidad de sus gastos financieros sean estos provenientes de partes vinculadas o no, hasta incluso de aquellos que provienen de paraísos fiscales63.

Adicionalmente, consideramos que esta ausencia de regulación se agravaría en tanto que existen argumentos para señalar que la Norma Anti-elusiva General (regulada en los párrafos del segundo a quinto de la Norma XVI del Código Tributario) no resultaría aplicable en este escenario.

Ello, debido a que de la lectura del D.L. 1424 y de la Exposición de Motivos se observa que la finalidad del legislador es perseguir la elusión tributaria en operaciones que generen deducciones de gastos financieros, esto es, una norma anti-elusiva específica para este tipo de operaciones.

En consecuencia, ante una eventual fiscalización donde se observe operaciones de fragmentación con la única intención de aprovecharse indebidamente de la excepción de la regla de subcapitalización, la Administración Tributaria no podrá someter dicha operación a un análisis de la Norma Anti-elusiva General, pues en virtud del principio de especialidad corresponderá aplicar lo establecido en el D.L. 1424 (donde como se mencionó) no existe regulación al respecto.

\section{d) Norma antielusiva específica, motivo económico y principio de causalidad}

En este punto debemos preguntarnos si la regla de limitación a la deducción de gastos financieros introducida por el D.L. 1424 tiene un fin antielusivo, corresponde que los contribuyentes tengan la posibilidad de probar en contrario.

Así, en vez de ser una regla objetiva que se configura con el solo cumplimiento del supuesto de hecho normativo, consideramos que debería de darse la posibilidad de que el contribuyente demuestre que las operaciones de financiamiento realizadas responden a motivos económicos válidos sin que medie un fin elusivo alguno.

63 Esto en el marco del Reporte Final de la Acción 4 donde se señala como grupo de riesgo a las empresas multinacionales y sus vinculadas y empresas que tienen subsidiarias en paraísos fiscales. 
Para tal efecto, coincidimos con Soler cuando señala que, tanto en las cláusulas generales como específicas, la prueba se sustenta en indicios, dada la dificultad de probar la intención de eludir del contribuyente y que en el supuesto de que la carga de la prueba se traslade al contribuyente, esta debe ser impuesta respetando un criterio de proporcionalidad 64 .

Así pues, somos de la opinión que, si bien para el contribuyente sería muy gravoso probar que no ha tenido una intención elusiva, sí podría ser factible que a partir de elementos objetivos pruebe que su decisión de realizar operaciones de financiamiento corresponde a un fin económico válido en conveniencia de sus actividades comerciales.

Cabe resaltar que aplicar el nuevo límite a la deducción de gastos financieros de manera inflexible no solo iría en contra de su propia finalidad de perseguir comportamientos elusivos, sino que incluso podría ir en contra del principio de causalidad. Ello en tanto que si el contribuyente tiene elementos suficientes para probar que los préstamos realizados están conducidos por motivos económicos (para generar renta o mantener su fuente productora), en aplicación de la regla de subcapitalización no podrán ser deducibles aun cuando cumplan con el referido principio.

Finalmente, resulta alarmante que en el contexto socioeconómico en el que nos encontramos, hasta el momento no haya pronunciamiento oficial que esclarezca si a los intereses generados por los créditos otorgados en el marco del programa Reactiva Perú -cuya finalidad consistió en asegurar la continuidad de la cadena de pagos ante el impacto del Covid-19- se encontrarán incluidos dentro del ámbito de aplicación de la norma de subcapitalización.

De ser tal respuesta afirmativa (como no parece de otra forma porque no se ha dictado una norma de excepción y una norma posterior no sería aplicable a los intereses hasta el momento devengados) generará una gran inconsistencia en las políticas públicas, pues mientras que por un lado se intenta rescatar a las empresas con préstamos a bajo costo, por otro lado, no se le permitirá deducir los intereses generados por dichos préstamos.

\section{e) Problemas en la regla aplicable a partir del 2021 y post Covid-19 y el arrastre de gasto financieros.}

El 2020 será recordado como el año de la proliferación del virus del Covid-19, donde no solo ha dejado una gran cantidad de pérdidas humanas a lo largo del mundo sino también estragos incalculables en materia económica.

Así las cosas, no cabe duda de que las empresas peruanas no generarán ingresos este año si no que incluso (lo más probable), aquellas registrarán pérdidas 
arrastrables para los siguientes periodos, como parte de una lenta recuperación económica.

En este contexto de necesaria atracción de capitales para la reactivación económica, llama poderosamente la atención que al día de hoy no existan proyectos de ley para modificar la regla de subcapitalización que será aplicable a partir del 2021. Ello en tanto que dicha norma prevé que no serán deducibles los intereses netos en la parte que exceden el $30 \%$ del EBIDTA del ejercicio anterior, esto es, del ejercicio 2020.

El EBITDA es definido por la misma norma como "Renta neta luego de efectuada la compensación de pérdidas más los intereses netos, depreciación y amortización". En consecuencia, para poder deducir gastos financieros, se debe tener una base de renta neta, hecho que, por las circunstancias actuales donde ninguna compañía está generando ingresos, no resultará posible.

Al respecto, consideramos que, incluso más allá de todos los problemas estructurales que hemos expuestos en los apartados anteriores, dada las circunstancias actuales que atraviesa la economía nacional, no corresponde aplicar un porcentaje del EBITDA como ratio para establecer el límite de intereses deducibles. Ello hará prácticamente nula la posibilidad de deducir intereses, situación que excede el fin antielusivo de la norma.

Cabe resaltar que el establecimiento del EBITDA como ratio para establecer el límite de gastos financieros deducibles resulta criticable incluso en circunstancias económicas normales, en tanto que dicha regla no prevé aquellos sectores que son muy volátiles como los mercados que están sujetos al valor internacional establecido para commodities o sectores cíclicos con fuertes inversiones en sus operaciones pre-operativas.

En este sentido se ha manifestado la profesora Johanna HEY quien sugiere que establecer un límite teniendo como referencia al EBITDA para la deducción de intereses podría dañar industrias con economías cíclicas y compañías en economías que se encuentren en crisis65.

Por último, aun cuando muchos especialistas aseguran que establecer una ratio en base al EBITDA resulta una metodología menos manipulable (en comparación de las reglas que utilizan el patrimonio o el activo de las empresas), en tanto no existe norma reglamentaria que complemente la breve definición propuesta por el D.L. 1424, quedan dudas sobre lo que se debe de considerar como parte de su determinación.

En otras palabras, aún en el supuesto que la utilización de un porcentaje del EBITDA como ratio para determinar el límite de intereses deducibles sea la "panacea" contra la elusión tributaria en las operaciones de financiamientos, la

65 HEY, Johanna. "Base Erosion and Profit Shifting and Interest Expenditure". En Bulletin for International Taxation, vol. 68, 2014, pp. 332-343. 
deficiente determinación legislativa de los conceptos amortización y depreciación, le restará eficacia para sus fines.

Si bien el problema se atenúa con la incorporación de la posibilidad de arrastrar los gastos financieros no deducidos a los siguientes cuatro ejercicios; lo cierto es que, como efecto inmediato, los costos de los financiamientos se verán incrementado, lo cual afecta directamente a las empresas que han visto incrementadas sus necesidades de endeudamiento.

Asimismo, es importante tomar en consideración que el Estado peruano tampoco ha seguido en su totalidad las recomendaciones de la OECD sobre el "carry forward". El Reporte Final de la Acción 4 recomienda dos tipos de arrastre:

1. Arrastre de los intereses que hubieran superado el límite establecido por la ratio.

2. Arrastre de la capacidad de intereses no utilizada (i.e. sí en un periodo los intereses deducidos son menores al límite determinado por la ratio, la diferencia podrá utilizarse en los ejercicios siguientes).

En definitiva, la norma peruana solo ha acogido el primer tipo de arrastre, con un límite de cuatro ejercicios. Justamente, cabe preguntarse, ¿cuál es la justificación para establecer dicho plazo? Si bien, la Acción 4 no recomienda un límite temporal específico, la legislación comparada demuestra que los países que han adoptado las ESR establecen un arrastre de intereses no deducidos por largos periodos. Inclusive, en el caso de la ESR italiana, se establece un arrastre sin límite temporal.

\section{Conclusiones}

i. La subcapitalización y el earnings stripping son fenómenos distintos.

ii. La subcapitalización originado en las ciencias económicas, puede ser de carácter real o nominal. La subcapitalización nominal (relevante para efectos tributarios) se define como la desproporción entre la deuda (pasivo) y el capital social de una sociedad, ocasionado por la decisión deliberada de los accionistas de financiarla a través de préstamos, en lugar de aportes de capital.

iii. En el ámbito tributario, generalmente, se asocia a la subcapitalización con la erosión de la base imponible de una sociedad mediante la deducción de intereses, a fin de determinar un menor impuesto a la renta.

iv. El earnings stripping, en cambio, es un tipo de planificación fiscal inherente a los grupos multinacionales (denominados en el presente trabajo como, MNEs), consistente en que una empresa (parte del grupo empresarial) erosiona su base imponible a través del pago de intereses a su matriz (o a otra empresa del grupo), obteniendo una ventaja fiscal. No obstante, este fenómeno es más amplio que la subcapitalización: (a) no se restringe al pago 
de intereses, pues se puede originar en el pago de regalías u otros pagos; $y$ (b) puede originarse en el financiamiento de terceros.

v. Así pues, las diferencias entre ambos conceptos son de tres tipos: (a) conceptual, (b) subjetiva (i.e., sujetos intervinientes) y, (c) objetiva (financiamientos que son objeto de la limitación).

vi. Tales diferencias se reflejan en la estructura, alcance y efectos de los mecanismos legislativos empleados por los Estados para combatirlos.

vii. Así pues, el mecanismo aplicado por excelencia para combatir la subcapitalización han sido las thin capitalization rules. Por naturaleza, las TCR sancionan con la recategorización de los intereses (como dividendos) provenientes de préstamos otorgados por partes vinculadas que excedan el límite calculado a través un ratio de endeudamiento sobre patrimonio.

viii. En contraste, el reciente mecanismo empleado para combatir el earnings stripping es la aplicación de las earnings stripping rules. Por regla general, las ESR establecen un límite general a la deducción de intereses (sin distinguir si provienen de partes vinculadas o de terceros) equivalente a un porcentaje de los ingresos de una sociedad (usualmente, se toma como referencia el EBITDA).

ix. No obstante, la legislación comparada demuestra que las diferencias conceptuales no siempre han sido plasmadas con exactitud. En efecto, los estados fueron modificando sus TCR incorporando características que no respondían, propiamente, a su naturaleza, si no a la de una ESR. Justamente, la última etapa de esta evolución es el reemplazo de las TCR por ESR. Esto es una muestra que, los estados progresivamente cambiaron el objeto de su lucha al earnings stripping en lugar de la subcapitalización.

x. El Perú incorporó por primera vez una TCR en 2001, la cual se mantuvo prácticamente - sin modificaciones hasta 2018. Justamente, dicha modificación introdujo modificaciones sustanciales a la referida norma. Dicha norma introdujo dos regímenes: (a) uno transitorio aplicable en los años 2019 y 2020; y, (b) uno definitivo aplicable a partir de 2021.

xi. Tales modificaciones reflejan la evolución que han tenido las TCR a nivel mundial. Precisamente, el régimen transitorio, mantiene en esencia una TCR, aunque con un ámbito de aplicación objetivo más amplio (i.e., incluye financiamientos otorgados por terceros), característica propia de una ESR. En cambio, el régimen definitivo, reemplaza dicha TCR por una ESR, estableciendo un límite general a la deducción de intereses del 30\% del EBITDA tributario.

xii. Es de notar que, las modificaciones introducidas en 2018 no responden a un análisis del contexto económico o jurídico-tributario de nuestro país, sino que responden a las recomendaciones de la OECD, quien a través de la 
¿Regla de subcapitalización o earnings stripping rule?: Análisis evolutivo y comparativo de las normas que limitan la deducción de gastos financieros y su enfoque en el Perú

Acción 4 del Proyecto BEPS recomendó a los países miembros adoptar una ESR en lugar de una TCR.

xiii. No obstante, las recomendaciones de la OECD fueron pensadas para las economías de los países miembro y, específicamente, para los miembros del G-20. Ciertamente, la economía de nuestro país no tiene las mismas características por lo que, la adopción de estas recomendaciones conlleva una serie de problemas estructurales.

xiv. De igual forma, la entrada en vigor del régimen definitivo a partir del 1 de enero de 2021 dada las circunstancias actuales que atraviesa la economía nacional tendrá más perjuicios que beneficios. En concreto, debido a la paralización de la economía ocasionada por el Covid-19 y posteriores medidas de aislamiento social obligatorio, significará un número significativo de empresas tengan pérdidas en los siguientes ejercicios. Por lo tanto, al poder aplicar el porcentaje del EBITDA tributario dichas empresas no podrán deducir gastos financieros. 